\title{
Melatonin Receptor Agonist Piromelatine Ameliorates Impaired Glucose Metabolism in Chronically Stressed Rats Fed a High-Fat Diet
}

\author{
Jun Zhou, Deng Wang, XiaoHong Luo, Xu Jia, MaoXing Li, Moshe Laudon, RuXue Zhang, \\ and ZhengPing Jia
}

\begin{abstract}
College of Pharmacy, Lanzhou University, Lanzhou, PR China (J.Z., M.X.L, R.X.Z, Z.P.J.); Xi'an Daxing Hospital, Shaanxi, PR China (D.W.); Lanzhou General Hospital of PLA, Lanzhou, PR China (J.Z., X.L., M.X.L, R.X.Z, Z.P.J.); Shanghai Institute of Endocrine and Metabolic Diseases, Shanghai Jiao Tong University School of Medicine, Shanghai, China (X.J.); and Drug Discovery, Neurim Pharmaceuticals Ltd., Tel-Aviv, Israel (M.L.)
\end{abstract}

Received July 19, 2017; accepted October 6, 2017

\begin{abstract}
Modern lifestyle factors (high-caloric food rich in fat) and daily chronic stress are important risk factors for metabolic disturbances. Increased hypothalamic-pituitary-adrenal (HPA) axis activity and the subsequent excess production of glucocorticoids (GCs) in response to chronic stress (CS) leads to increases in metabolic complications, such as type 2 diabetes and insulin resistance $(\mathrm{IR})$. Melatonin (MLT), which protects several regulatory components of the HPA axis from GC-induced deterioration, might improve glucose homeostasis. Piromelatine is a melatonin receptor-1/melatonin receptor-2 (MT1/MT2) agonist with high affinity for MLT receptors and a longer duration of action than MLT. The objective of the present study was to explore the potential effects of piromelatine on glucose and lipid metabolism and insulin sensitivity in rats with IR induced by a high-fat diet
\end{abstract}

combined with CS (CF). The results showed that piromelatine prevented the suppression of body weight gain and energy intake induced by CF and normalized CF-induced hyperglycemia and homeostasis model assessment-IR index, which suggests that piromelatine prevented whole-body IR. Piromelatine also prevented CF-induced dysregulation of genes involved in glucose and lipid metabolism, including proinflammatory cytokines, in adipose tissue. In addition, piromelatine also attenuated CF-induced excess free corticosterone release, increased glucocorticoid receptor expression, and decreased $11 \beta$-hydroxysteroid dehydrogenase-1 expression, suggesting that piromelatine might ameliorate impaired glucose metabolism and prevent IR by normalizing HPA-axis functions. In conclusion, piromelatine might be a novel therapeutic agent for glucose intolerance and IR.

\section{Introduction}

The prevalence of type 2 diabetes mellitus (T2DM) has been increasing rapidly over the last two decades, and modern lifestyle factors, including a hypercaloric diet and social and work pressure (psychologic stress), are thought to be responsible for this increase (Stumvoll et al., 2005; Hansen et al., 2010; Tirabassi et al., 2016). It is clear that chronic stress and endocrine stress responses are significantly associated with glucose intolerance, insulin resistance (IR), and diabetes mellitus (Siddiqui, et al., 2015). It has been reported that stressed people often reach for "comfort" food, which includes palatable food rich in carbohydrates and fat (Fachin et al.,

This work was supported by the National Natural Science Foundation of China (nos. 81173620 and 30772773). Authors declare that they have no conflicts of interest.

https://doi.org/10.1124/jpet.117.243998.
2008). A high-fat diet (HFD) might lead to obesity and exacerbate metabolic disorders.

The body responds to stress-induced homeostatic challenges by activating the hypothalamic-pituitary-adrenal (HPA) axis (Tirabassi et al., 2016). Chronic stress (CS) might impair the feedback mechanisms of the HPA axis that return these hormonal systems to normal, resulting in chronically elevated levels of glucocorticoids (GCs), catecholamines, and inflammatory markers (Joseph and Golden, 2017). As the final output of the HPA axis, the release of GCs from the adrenal glands serves to oppose insulin-mediated energy storage, in part by suppressing insulin secretion (Dinneen et al., 1993). By inducing a transient state of IR, excessive amounts of GCs restrain hepatic glucose production and regulate glucose delivery to peripheral tissues, thereby resulting in hyperglycemia (Rizza et al., 1982; Björntorp and Rosmond, 1999, 2000; Björntorp, 2001; Patterson and Abizaid, 2013).

ABBREVIATIONS: $11 \beta \mathrm{HSD} 1,11 \beta$-hydroxysteroid dehydrogenase-1; CF, high-fat diet combined with chronic stress; CORT, corticosterone; CS, chronic stress; FFA, free fatty acid; GCs, glucocorticoids; GLUT-4, glucose transporter type-4; GR, glucocorticoid receptor; HDL-C, high-density lipoprotein cholesterol; HFD, high-fat diet; HOMA, homeostasis model assessment; HPA, hypothalamic-pituitary-adrenal; IL-6, interleukin-6; IPGTT, intraperitoneal glucose tolerance test; IR, insulin resistance; ITT, insulin tolerance test; LDL-C, low-density lipoprotein cholesterol; MLT, melatonin; MT1/MT2, melatonin receptor-1/melatonin receptor-2; PMT-H, high-dose piromelatine-treated CF group; PPAR- $\gamma$, peroxisome proliferatoractivated receptor- $\gamma$; QR2, quinone reductase II; ROR/RZR, retinoid orphan receptors/retinoid Z receptors; T2DM, type 2 diabetes mellitus; qPCR, quantitative polymerase chain reaction; RT, reverse transcription; TC, total cholesterol; TG, triglyceride; TNF- $\alpha$, tumor necrosis factor- $\alpha$. 
Therefore, counteracting HPA axis hyperactivity is a challenge in stress-related metabolic disorders.

Melatonin (5-methoxy- $N$-acetyltryptamine) (MLT) is a circulating hormone primarily produced and secreted by the pineal gland that acts as a major synchronizer of endocrine rhythms (Detanico et al., 2009) and is a homeostatic regulator or pharmacological buffer of the effects of stress (Kopp et al., 1999). Several studies have found that MLT administration is associated with diminished overall corticosterone (CORT) secretion and increased sensitivity to GC feedback (Houdek et al., 2015), which suggests that MLT might serve as a physiologic regulator of the HPA axis (Konakchieva et al., 1997). Studies have suggested that chronic MLT treatment might protect several regulatory components of the HPA axis from GC-induced deterioration and might improve glucose homeostasis not only in pinealectomized rats that display glucose intolerance but also in rats with IR (Konakchieva et al., 1998; Sartori et al., 2009; Shieh et al., 2009; Kitagawa et al., 2012).

As a melatonin receptor-1/melatonin receptor-2 (MT1/MT2) agonist, piromelatine [ $N$-(2-(5-methoxy-1H-indol-3-yl)ethyl)4-oxo-4H-pyran-2-carboxamide] displays high affinity for MLT receptors and has a longer duration of action than MLT (Yalkinoglu et al., 2010; Carocci et al., 2014), as shown in Table 1. The chemical structures of MLT and piromelatine are presented in Fig. 1. Studies have shown that piromelatine functions in a manner similar to MLT and promotes sleep (Laudon et al., 2008), alleviates lipid peroxidation, protects mitochondria from myocardial ischemia/reperfusion injury (Yu et al., 2014), exerts neuroprotective effects against brain ischemia (Buendia et al., 2015), exhibits antidepressant and anxiolytic activities (Tian et al., 2010), inhibits body-weight gain, and increases sensitivity to insulin in obese rodent models (She et al., 2009). In our research, we explored the potential effects of piromelatine on glucose metabolism and insulin sensitivity in rats that were chronically stressed and fed an HFD (CF).

\section{Materials and Methods}

\section{Experimental Animals}

Adult male Wistar rats $(200 \pm 20 \mathrm{~g})$ were provided by the Animal Facility Center of Lanzhou University (approval no. 14-006), China.

TABLE 1

Gene-specific primers used in this study

\begin{tabular}{lc}
\hline \multicolumn{1}{c}{ Gene } & Primer sequences (5' to 3') $^{\prime}$ \\
\hline$\beta$-actin & Forward: GGAGATTACTGCCCTGGCTCCTA \\
GR & Reverse: GACTCATCGTACTCCTGCTTGCTG \\
& Forward: AAGGTTAGCAGAGGGAGGCTTT \\
$11 \beta$-HSD1 & Reverse: GAAGGGTGGGGAGGATTAGTG \\
& Forward: CCCATGTGGTATTGATGCAAG \\
Adiponectin & Reverse: AGAGGCTGCTCCGAGTTCAAG \\
& Forward: GCCTATCCACAAAGTCCAGGATG \\
Leptin & Reverse: CCCGGGAATGAAGTCCAAAC \\
& Forward: GCCTATCCACAAAGTCCAGGATG \\
TNF- $\alpha$ & Reverse: CCCGGGAATGAAGTCCAAAC \\
& Forward: ATACACTGGCCCGAGGCAAC \\
IL-6 & Reverse: CCACATCTCGGATCATGCTTTC \\
& Forward: ATTGTATGAACAGCGATGATGCAC \\
PPAR- $\gamma$ & Reverse: CCAGGTAGAAACGGAACTCCAGA \\
GLUT-4 & Forward: TGCAGCAGGTTGTCTTGGATG \\
& Reverse: GGAGCCTAAGTTTGAGTTTGCTGTG \\
& Forward: TATTTGCCGTCCTCCTGCTT \\
& Reverse: TGGGTTTCACCTCCTGCTCT \\
\hline
\end{tabular}
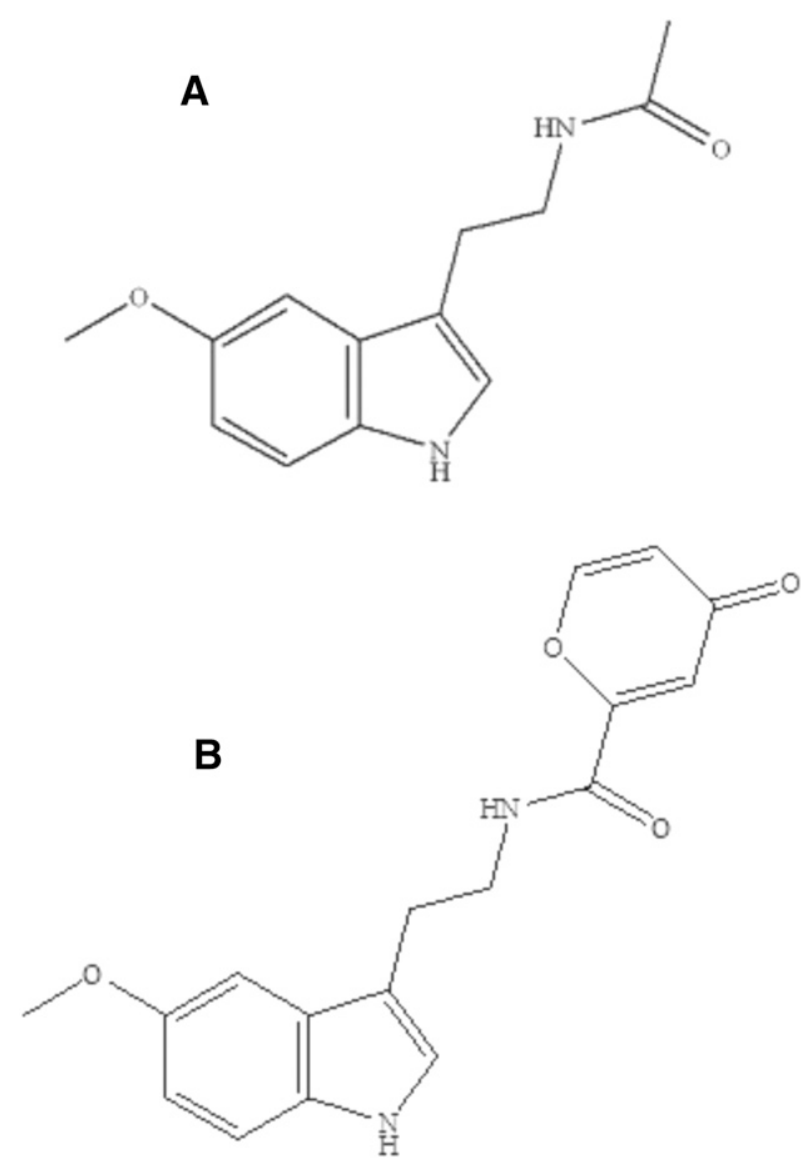

Fig. 1. Structure of MLT and piromelatine Chemical structures of MLT (A) and piromelatine (B).

The animals were allowed to acclimatize for at least 6 days before the experiments and were housed under controlled illumination (12-hour light/12-hour dark cycle; lights on from 7:00 AM to 7:00 PM) and ambient temperature $\left(\sim 20-24^{\circ} \mathrm{C}\right)$ and humidity $(40-60 \%)$ with food and water ad libitum. Animal welfare and experimental procedures complied with the guidelines of the Gansu Experimental Animal Center (Gansu, China) and were officially approved by the Ministry of Health, P. R. China, in accordance with NIH guidelines (2002). The laboratory procedures were carried out under the permission and surveillance of the PLA Lanzhou General Hospital Ethics Committee.

\section{Reagents}

MLT and piromelatine were synthesized and provided by Neurim Pharmaceuticals Ltd. (Tel-Aviv, Israel). The compounds were dissolved in ethanol and diluted in sterile saline to obtain a final ethanol concentration of $0.02 \%$. All drugs were administered by oral gavage in a volume of no more than $10 \mathrm{ml} / \mathrm{kg}$.

\section{Experimental Designs}

Experiment I: Effect of an HFD and Stress on Rat Metabolism. Adult male Wistar rats $(200 \pm 20 \mathrm{~g})$ were randomly divided into four experimental groups: a control group was fed commercial standard chow; an HFD group was fed high-fat chow instead of commercial standard chow; a CS group was fed the same diet as the control group and exposed to chronic, unpredictable stress; and a CF group was fed the same diet as the HFD group and exposed to chronic stress.

The standard rat chow diet (13 kJ/g; 5\% saccharides; $12.5 \%$ fat) contained $53.1 \%$ carbohydrates and $4 \%$ lipids. The HFD $(24 \mathrm{~kJ} / \mathrm{g} ; 25 \%$ 
saccharides; $62 \%$ fat) consisted of $40 \%$ standard diet, $20 \%$ edible lard, $5.5 \%$ carbohydrates, $35 \% \mathrm{egg}(\mathrm{w} / \mathrm{w})$, and $0.9 \% \mathrm{NaCl}$. The chows were prepared by the Animal Experimental Center of Lanzhou General Hospital, PLA, China.

The stress methods were improved on the basis of the report by Zardooz et al. (2006) and included the following stressors: 1) absorbent gauze wrap secured with tape, 2) restraint in a polyvinyl chloride tube $(\mathrm{L}=25 \mathrm{~cm}$, i.d. $=5 \mathrm{~cm})$ closed at both ends, 3$)$ immobilization on a board with tape, and 4) tail suspension. The stress protocol involved exposure to one of the first three restraint stressors for a 2-hour period from 9:00 to 11:00 AM or to the fourth stressor for 30 minutes from 9:00 to 9:30 AM once daily. The animals were exposed in turn to one of the above stressors once a day and then returned to the animal facility 15 minutes after the stress exposure to minimize disturbance to the control group.

Body weight and fasting blood glucose (6-hour) were measured weekly. The insulin tolerance test (ITT, $0.5 \mathrm{IU} / \mathrm{kg}$ ) was performed at day 21 , and the intraperitoneal glucose tolerance test (IPGTT, 2.5 $\mathrm{g} / \mathrm{kg}$ ) was performed at day 28 . At the end of the 33 days of treatment, animals were fasted for 12 hours and then killed via decapitation. Their blood was sampled to perform lipid, free fatty acid (FFA), CORT, insulin, glucagon, and leptin measurements, and their liver and muscle tissues were sampled for glycogen assays.

Experiment II: Induction of Insulin Resistance and Experimental Design. After an acclimation period, the rats were randomly divided into five groups: a normal control group (control group, sterile saline $2 \mathrm{ml} / \mathrm{kg}$ ), a chronically stressed and HFD-fed group (CF group, sterile saline $2 \mathrm{ml} / \mathrm{kg}$ ), an MLT-treated CF group (MLT group, $20 \mathrm{mg} / \mathrm{kg}$ ), a IPGTT group, $10 \mathrm{mg} / \mathrm{kg}$ ), and a high-dose piromelatinetreated CF group (PMT-H group, $20 \mathrm{mg} / \mathrm{kg}$ ). The treated rats were administered drugs by gavage from 8:00 to 9:00 AM, whereas the control group and CF group were administered an equal volume of sterile saline. After drug administration, the control group was fed a standard diet and was not exposed to any stressors, whereas the other experimental groups were fed an HFD and simultaneously stimulated with chronic stress for 33 days.

Food intake and water consumption were recorded daily. Body weight and fasting blood glucose (6-hour) were measured weekly. The IPGTT $(2.5 \mathrm{~g} / \mathrm{kg})$ was performed at day 28 . At the end of the treatment, the animals were fasted for 12 hours and then killed via decapitation. Whole blood and tissue samples were collected for subsequent analyses.

\section{Measurement of Body Weight, Food Intake and Water Consumption}

Body weights were monitored weekly in the morning. The rats were fed daily on a precise feeding schedule (at 8:00 AM) with equal amounts of food and water. Each rat was provided with approximately $30 \mathrm{~g}$ of chow and $180 \mathrm{~g}$ of water. Food intake and water consumption were measured by subtracting the remaining food and water before feeding. The relative food intake was calculated as grams of daily food intake per $100 \mathrm{~g}$ body weight, and the relative water consumption was calculated as grams of daily water intake per $100 \mathrm{~g}$ body weight.

\section{Measurement of Fasting Blood Glucose Levels}

The fasting blood glucose level (after 6 hours of fasting) was measured weekly on day (d)0, d7, d14, d21 and d28. Fasting blood glucose levels were estimated using commercially available glucose kits whose basis is the glucose oxidase method according to the manufacturer's instructions. The results are expressed as millimoles per liter of plasma.

\section{IPGTT}

All animals were fasted for 6 hours and then injected intraperitoneally with a single dose of $50 \%$ glucose at a concentration of $2.5 \mathrm{~g} / \mathrm{kg}$ body weight. Blood samples were collected from each group just before glucose administration ( 0 minutes) and at 30, 60, and 120 minutes after glucose administration. Plasma glucose levels were determined by the glucose oxidase method. The results are expressed as millimoles per liter.

\section{ITT}

All animals were fasted for 6 hours and then injected intraperitoneally with a single dose of insulin at a concentration of $0.5 \mathrm{IU} / \mathrm{kg}$ body weight. Blood samples were collected from each group immediately before insulin administration ( 0 minutes) and at 30, 60, and 120 minutes after insulin administration. Plasma glucose levels were determined via the glucose oxidase method and are expressed as millimoles per liter plasma.

\section{Plasma Lipid Determination}

Plasma was collected after rat sacrifice as described above. The cholesterol oxidase-peroxidase-4-aminoantipyrine-phenol (CHODPAP) method was used to determine the plasma total cholesterol (TC) levels, and the glycerol phosphate oxidase-peroxidase-4aminoantipyrine-phenol (GPO-PAP) method was used to determine the plasma triglyceride (TG) levels according to the instructions provided with the kit. The plasma high-density lipoprotein cholesterol (HDL-C) levels were determined by the phosphotungstic acid-magnesium ${ }^{2+}\left(\mathrm{PTA}-\mathrm{Mg}^{2+}\right)$ precipitation method, whereas the plasma low-density lipoprotein cholesterol (LDL-C) levels were determined by the polyethylene-sulfuric acid-precipitation (PSAP) method according to instructions provided with the kit. Free fatty acids (FFAs) were measured via the biscyclohexanoneoxaiyldihydraone coloration method using commercial assay kits according to the manufacturer's directions. All values are expressed as millimoles per liter.

\section{Measurement of Plasma Glucagon, Insulin, and Leptin Levels and the Homeostasis Model Assessment-IR Index}

The glucagon, insulin, and leptin levels in frozen plasma samples were measured using Rat glucagon, insulin, leptin Enzyme Immunoassay Kits. The IR index estimated by the homeostasis model assessment (HOMA) was calculated using relationships between the blood glucose and insulin levels according to the following formula (Matthews et al., 1985):

$$
\begin{aligned}
& \text { HOMA-IR }=[\text { fasting serum insulin }(\mathrm{mIU} / \mathrm{L}) \\
& \times \text { fasting blood gulcose }(\mathrm{mmol} / \mathrm{L})] / 22.5
\end{aligned}
$$

\section{Measurement of Glycogen Levels}

The glycogen contents of liver and skeletal muscles were measured using the anthrone-sulfuric acid colorimetric assay method as previously established (Sadasivam and Manickam, 1996), according to the manufacturer's instructions. The amounts of glycogen in the tissue samples are expressed as milligram glucose per gram tissue (wet weight).

\section{Measurement of Plasma CORT and 24-Hour Urinary CORT}

Using commercially available radioimmunoassay kits for rat CORT, the plasma and 24-hour urinary levels of CORT were estimated according to instructions provided with the kit. The results are expressed as nanomoles per liter.

\section{Total RNA Isolation and Quantitative Real-Time Polymerase Chain Reaction}

For mRNA analysis, total RNA was extracted from tissues using a MiniBEST Universal RNA Extraction Kit (Takara Bio Inc., Shiga, Japan), and cDNA was synthesized using a PrimeScript RT Reagent Kit (cDNA Synthesis Premix; Takara Bio Inc.). The cDNA was 
amplified by quantitative PCR (qPCR) with SYBR Premix Ex TaqII (qPCR Premix; Takara Bio Inc.). The thermocycling conditions were as follows: initial denaturation at $95^{\circ} \mathrm{C}$ for 3 minutes followed by 40 cycles of denaturation at $95^{\circ} \mathrm{C}$ for 3 seconds, annealing at $72^{\circ} \mathrm{C}$ for 30 seconds, and extension at $60^{\circ} \mathrm{C}$ for 30 seconds. The $\beta$-actin gene served as the internal control. The cycle threshold $(\mathrm{Ct})$ value obtained for each target gene was normalized to that of the $\beta$-actin gene using the formula $2^{-\Delta \Delta \mathrm{Ct}}$. The relative quantity of each gene was expressed as the fold change with respect to the control after normalization to $\beta$-actin. The gene-specific primers used in this study to amplify the glucose and lipid metabolism-related genes glucocorticoid receptor (GR), 11 $\beta$-hydroxysteroid dehydrogenase (11 $\beta$-HSD) 1 , adiponectin, leptin, tumor necrosis factor (TNF)- $\alpha$, interleukin (IL)-6, peroxisome proliferator-activated receptor (PPAR)- $\gamma$, and glucose transporter type (GLUT)-4 are shown in Table 1.

\section{Western Blot Analysis}

The relative protein expression of GR and 11 $\beta$-HSD1 in adipose tissue were determined according to methods described previously (Zhou et al., 2017). Tissues were washed with ice-cold phosphatebuffered saline. After homogenization and centrifugation, the proteins in the supernatant were quantified using a Pierce BCA protein assay kit (Thermo Fisher Scientific, Waltham, MA) according to the manufacturer's instructions. Total proteins $(20 \mathrm{mg})$ were separated by $4-12 \%$ SDS-PAGE and then transferred to polyvinylidene difluoride membranes (Millipore, Bedford, MA). The membranes were blocked with 5\% skim milk for 1 hour and then incubated with anti-GR or anti-11 $\beta$-HSD1 polyclonal antibodies (diluted 1:1000; Abcam, Cambridge, MA) at $4^{\circ} \mathrm{C}$ overnight. The membranes were washed three times with Tris-buffered saline containing Tween 20 and then incubated for 60 minutes with the corresponding horseradish peroxidase-conjugated goat anti-mouse antibody (diluted 1:5000; ZSGB-BIO, Beijing, China). The proteins were then visualized with electro-chemiluminescence Western blotting detection reagents (Beijing Solarbio Science \& Technology Co., Ltd., Beijing, China) according to the manufacturer's instructions. The protein bands were evaluated using a Tanon-4200SF Biomolecular Imager (Tanon Science and Technology Co., Shanghai, China) and analyzed with ImagePro Plus 6.0 (Media Cybernetics, Silver Spring, MD). All signals were normalized to that of the housekeeping protein $\beta$-actin.

\section{Statistical Analyses}

Statistical analyses were conducted with GraphPad Prism 5.0 Software, and the results are expressed as the means \pm S.D. Statistical significance was determined using one-way analysis of variance with Dunnett's post-hoc analyses or two-way analysis of variance with Bonferroni post-hoc analyses according to the number of comparisons and variables. IPGTT and ITT data converted to area under the curve (AUC) values using one-way analysis of variance with Dunnett's post-hoc analyses. When an effect was statistically significant $(P<0.05, P<0.01)$, mean comparisons were performed.

\section{Results}

Effects of CS and HFD on Metabolic Parameters in Rats. To assess the role of CS on the metabolic profile of rats exposed to an HFD, metabolic parameters were studied in the groups subjected to CS/HFD.

The body weights of the control rats increased progressively throughout the 5-week experiment. The HFD showed a larger elevation in body weight compared with itself in week 0 , but did not differ from control rats significantly.

Compared with control group, CS challenge produced significant decreases in body weight gain from the third week to the end of the experiment $(P<0.05)$, addition of the HFD to the CS challenge decreased the body weight gain from the second week to the end of the experiment $(P<0.05)$ (Fig. 2A).

Both the CS and CF groups exhibited higher glucose levels compared with their respective control $(P<0.05)$. From the second week to the end, CF rats increased the fasting glucose concentration compared with that in week 0 . There was no statistically significant difference between the control and HFD groups (Fig. 2B).

Glucose tolerance was assessed via the IPGTT on day 28. After the 4-week experiment, compared with the normal group, the HFD or CS challenges alone significantly increased the glucose levels by $41.54 \%(P<0.05)$ and $39.23 \%(P<0.05)$, respectively, at 30 minutes, and increased the area under the curve (AUC) significantly $(P<0.05)$. Addition of the HFD to the CS challenge increased the blood glucose concentration by $71.54 \%(P<0.01)$ and $48.35 \%(P<0.05)$ at 30 and 60 minutes after glucose overload, respectively, and increased the AUC significantly $(P<0.01)$ (Fig. $2, \mathrm{C}$ and $\mathrm{D})$.

Insulin sensitivity was assessed via the ITT on day 21 . Compared with the normal control group, the CS, HFD, and $\mathrm{CF}$ groups displayed hyperglycemia, showing increases in glucose concentrations of $17.24 \%(P<0.05), 17.24 \%(P<0.05)$, and $31.03 \%(P<0.05)$, respectively, at 30 minutes and of $35 \%$ $(P<0.05), 30 \%(P<0.05), 40 \%(P<0.05)$, respectively, at 60 minutes after insulin injection $(0.5 \mathrm{IU} / \mathrm{kg})$. Only $\mathrm{CF}$ increased AUC significantly $(P<0.05)$ (Fig. $2, \mathrm{E}$ and F).

There was no statistically significant difference between the control and HFD groups in the liver and muscle glycogen contents. However, the CS and CF groups exhibited decreased glycogen storage in the liver and muscle tissues $(P<0.05)$ (Fig. 2, G and H).

The statistical analysis showed that significant effects of the CS and HFD treatment were observed on lipid metabolism. Both HFD-fed groups exhibited increased TG levels compared with the groups maintained on the standard diet $(P<0.05)$. Compared with the control animals, elevated TC concentrations were found in the blood of the CS group and both HFDfed groups $(P<0.05)$ (Fig. 2I). In addition, both stress groups showed increased FFA levels compared with the controls, but the FFA levels did not differ between the control and HFD group $(P<0.05)$ (Fig. 2J).

The blood hormone data are shown in Fig. $2 \mathrm{~K}-\mathrm{N}$. There was a trend toward an increase in the CORT, insulin, glucagon, and leptin concentrations in the groups that were subjected to CS/HFD; however, no statistically significant differences were observed.

Effect of Piromelatine on Body Weight, Food Intake, Water Consumption and Fasting Blood Glucose in Chronically Stressed Rats Fed an HFD. To examine the effects of piromelatine on chronically stressed rats fed an HFD, we monitored the body weights of rats weekly in the control, CF, MLT (CF + $20 \mathrm{mg} / \mathrm{kg}$ MLT), PMT-L (CF + $10 \mathrm{mg} / \mathrm{kg}$ piromelatine), and PMT-H (CF $+20 \mathrm{mg} / \mathrm{kg}$ piromelatine) groups over a 4-week treatment period. Compared with the control group, the weekly weight gains of the CF rats from the second week to the end of the experiment were reduced by $5.48 \%, 8.65 \%(P<0.05)$, and $13.51 \%(P<0.01)$, and these reductions in weight gain were attenuated by piromelatine (Fig. 3A).

The daily food intake and water consumption of the experimental rats were monitored to further examine the effects of piromelatine. CF animals exhibited significantly 
A

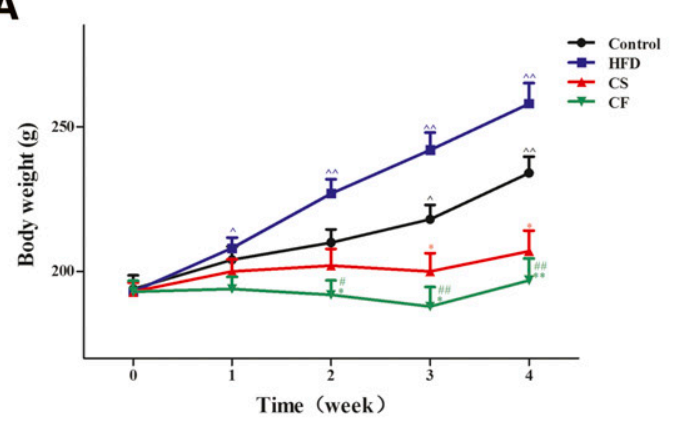

C

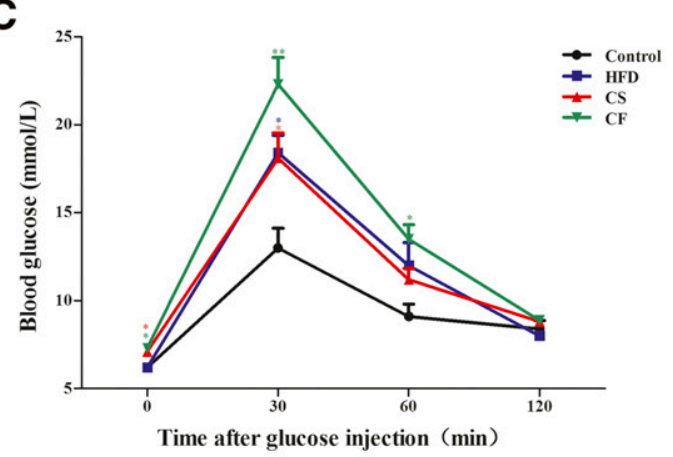

E

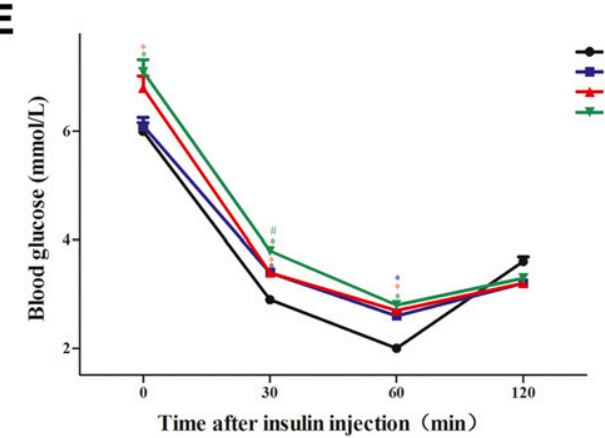

I

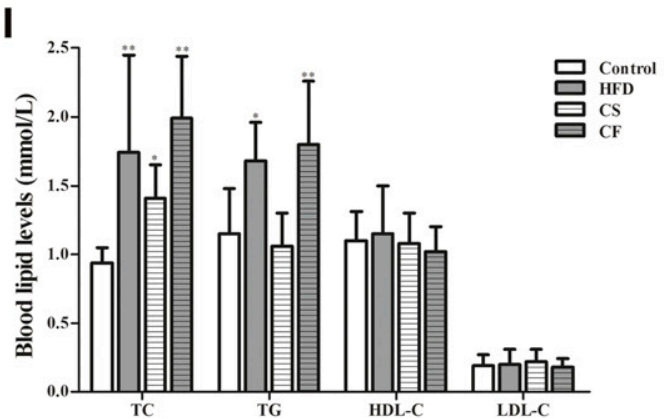

L

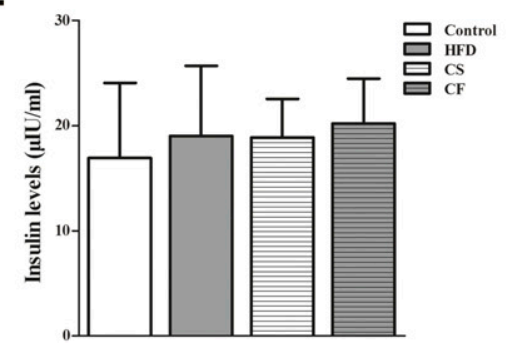

B

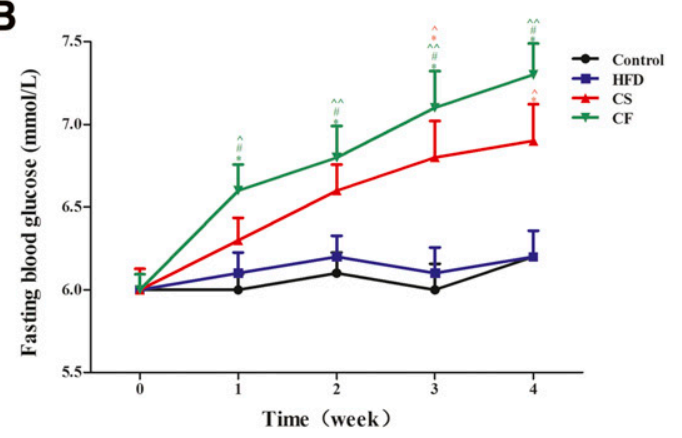

D

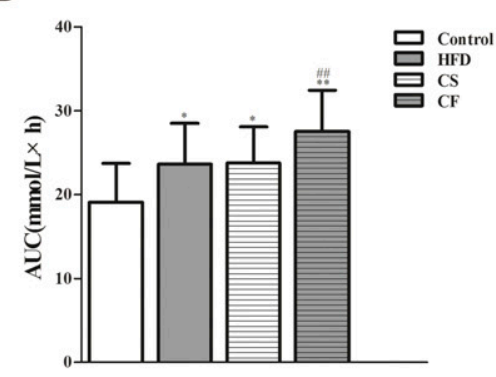

G
F

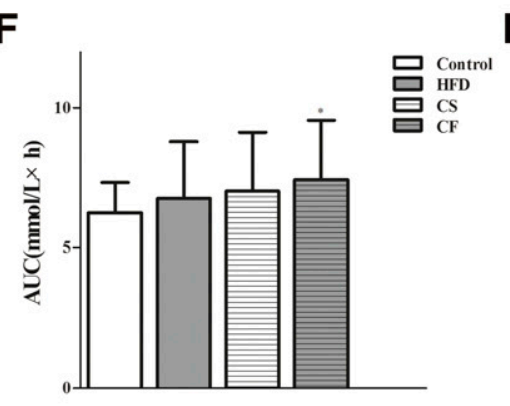

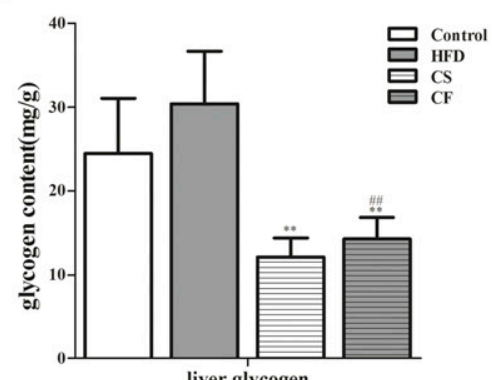

H

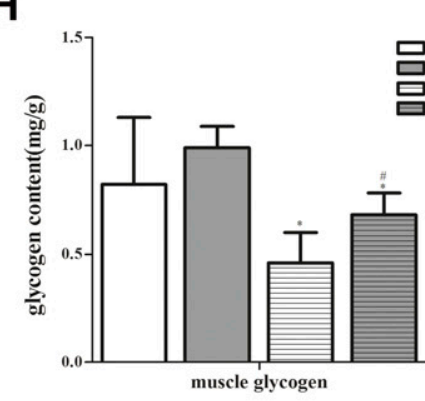

K

J
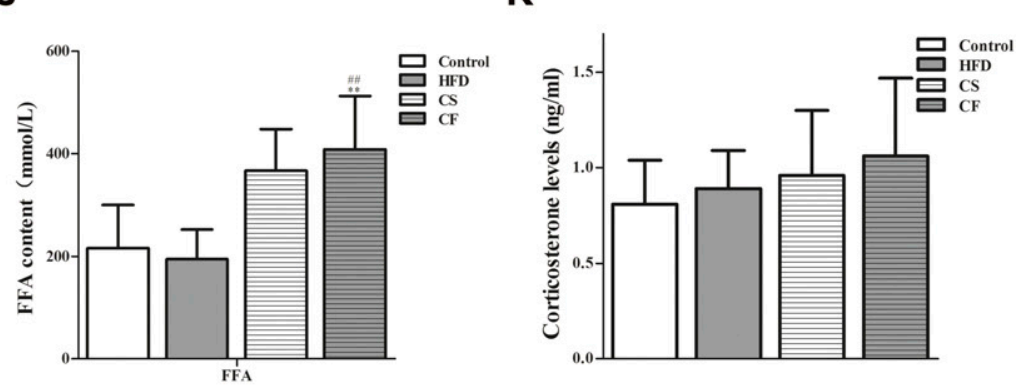

M
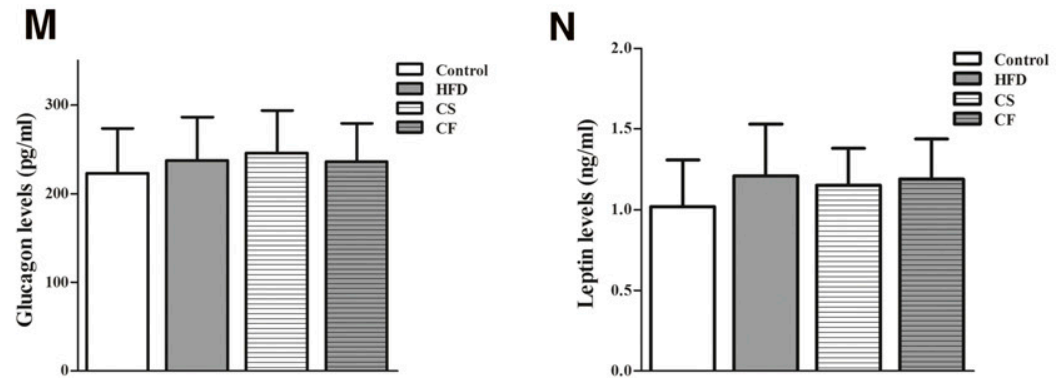
lower food intake levels $(P<0.01)$ and tended to have increased water consumption compared with control animals from the second week to the end of the experiment. Piromelatine treatment significantly increased the food intake of CF rats $(P<0.05)$ on the second week and resulted in higher energy consumption throughout the experiment (Fig. 3B). MLT tended to reduce the increased water consumption caused by CF throughout the 4 -week period, but piromelatine had no effect on water consumption (Fig. 3C). These results suggest that piromelatine attenuates the lower food intake in CF rats.

To determine whether MLT and piromelatine can lower the increased blood glucose levels in CF rats, we measured the fasting blood glucose levels in each group of rats (Fig. 3D). Compared with the control animals, CF rats exhibited higher fasting blood glucose levels from the second week to the fourth week of the experiment $(P<0.05)$. However, in the first and second weeks of treatment, MLT administration lowered the blood glucose levels by $15.74 \%$ and $13.81 \%$, respectively $(P<$ 0.05 and $P<0.01$ vs. CF animals), whereas PMT-H treatment decreased the blood glucose levels by $20.98 \%$ and $11.23 \%(P<$ 0.01 and $P<0.05$ vs. CF animals) in the third and fourth weeks, respectively. These results indicate that piromelatine attenuates many of the basic physiologic changes caused by chronic stress and an HFD in rats.

Effect of Piromelatine on IPGTT in Chronically Stressed Rats Fed an HFD. To further examine the effects of piromelatine on glucose homeostasis, we performed an IPGTT to assess the tolerance of rats after a bolus injection of glucose (2.5 g/kg i.p.). After being challenged with an oral bolus of glucose, CF animals showed a higher glucose excursion than normal control animals, whereas treatment with PMT-H inhibited the increase in blood glucose level by $17.67 \%(P<0.01), 11.07 \%, 7.37 \%$, and $10.36 \%(P<0.05)$ at 0 , 30,60 , and 120 minutes, respectively (Fig. 3E), as indicated by the approximately $13.8 \%$ drop in the AUC (Fig. 3F), suggesting improved insulin sensitivity. The rats treated with MLT or a low dose of piromelatine did not exhibit significant differences in glucose tolerance compared with the CF group.

Effect of Piromelatine on Insulin Level and HOMA-IR in Chronically Stressed Rats Fed an HFD. To determine whether piromelatine can improve IR in CF rats, we measured the fasting insulin levels and calculated the IR index in each group of rats (Fig. 3, G and H). The mean IR values calculated at the end of the study by the HOMA were higher in the CF group than in the control group. After 4 weeks of treatment, CF rats that received a high dose of piromelatine had a significantly reduced IR index compared with the CF group $(P<0.05)$. These results suggest that piromelatine attenuates IR and improves the insulin sensitivity caused by chronic stress and an HFD in rats.
Effect of Piromelatine on Blood Lipids in Chronically Stressed Rats Fed an HFD. To determine whether MLT and piromelatine affect the blood lipid content in CF rats, we assessed the levels of different types of lipids. After 4 weeks, the levels of TC, TG, and LDL-C were elevated by $21.13 \%(P<0.01), 39.84 \%(P<0.01)$, and $15.79 \%$, respectively, in the CF group compared with the control group. Treatment with MLT decreased the TC levels significantly by $16.37 \%(P<0.05)$, and piromelatine tended to decrease the TC, TG, and LDL-C levels, although the differences did not reach statistical significance (Fig. 4).

Effect of Piromelatine on CORT Concentrations in Chronically Stressed Rats Fed an HFD. GCs are a type of steroid hormone naturally produced in the adrenal cortex upon activation of the HPA axis, and their analog in rats is CORT. To assess the effects of piromelatine on CORT levels, we measured the total CORT concentrations (free and bound in circulation) in plasma and the CORT levels (average free in circulation) in urine. There were no significant differences in the plasma concentrations of CORT in CF rats compared with normal rats, whereas the administration of MLT and PMT-H reduced the plasma CORT by $35.74 \%$ and $42.96 \%$, respectively (both $P<0.05$ ). In contrast, the urinary CORT in CF rats was elevated by $28.94 \%(P<0.05)$ compared with that in control rats, and low-dose and high-dose piromelatine administration reduced urinary CORT by $13.38 \%(P<0.05)$ and $16.55 \%(P<$ 0.01) (Fig. 5, A and B). MLT also decreased the urinary CORT by $10.50 \%$, but the difference was not statistically significant. These results suggest that piromelatine decreases both the total and free CORT concentrations in rats subjected to stress and a high-fat intake.

Effect of Piromelatine on GR and 11 $\beta$ HSD-1 mRNA Expression in Adipose Tissue in CF Rats. $11 \beta$ HSD1 is a prereceptor enzyme that intracellularly converts less active GCs to a more active form (Stimson and Walker, 2013). The active GCs then mediate their effects through the GR, an intracellular hormone-activated transcriptional regulator (Kovačević et al., 2016).

To further investigate the prereceptor GC metabolism of HPA-axis regulators in chronically stressed rats fed an HFD, we assayed GR and $11 \beta$-HSD1 mRNA expression in adipose tissue by qPCR. The mRNA expression of $11 \beta$-HSD1, the key player in intracellular cortisol metabolism, was elevated in $\mathrm{CF}$ rats by $42.16 \%(P<0.01)$ compared with control rats, which is consistent with active CORT release. CF also reduced GR mRNA expression by $9 \%(P<0.05)$, indicating inhibition of HPA-axis feedback (Fig. 5C). 11 $\beta$-HSD1 mRNA expression was decreased by both MLT and piromelatine treatment $(P<$ 0.01 vs. CF), and GR mRNA expression was increased by PMT-H treatment $(P<0.01$ vs. CF), whereas MLT treatment had no significant effect on GR expression. These results indicate that piromelatine induces changes in gene expression

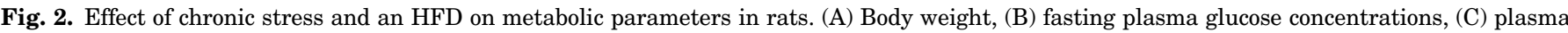

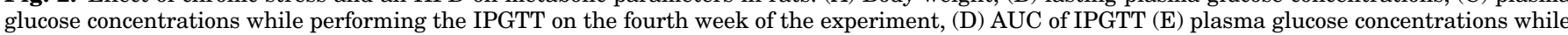

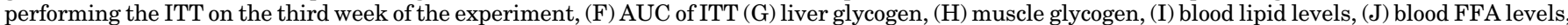

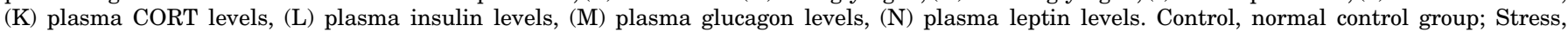

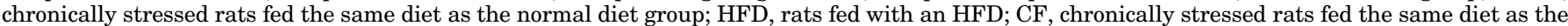

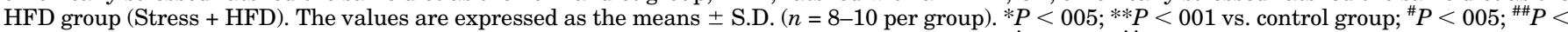

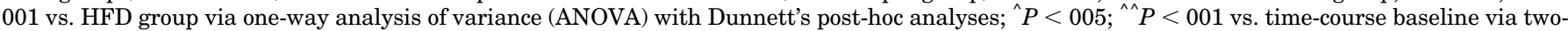
way ANOVA with Bonferroni post-hoc analyses. 
A

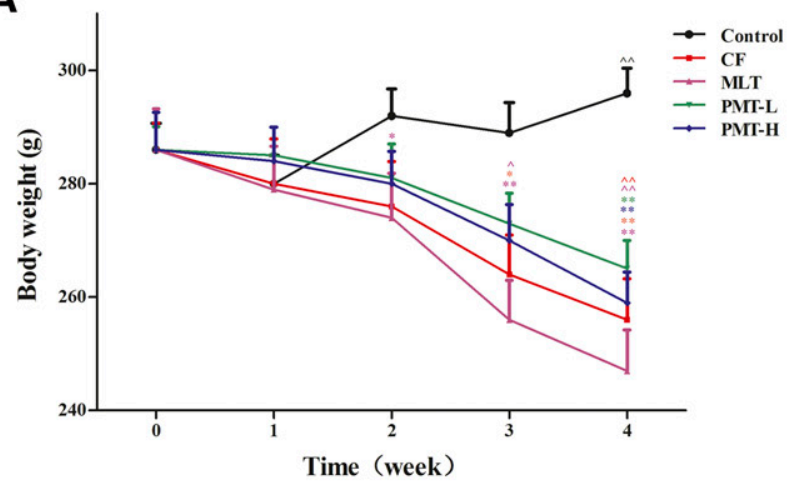

C

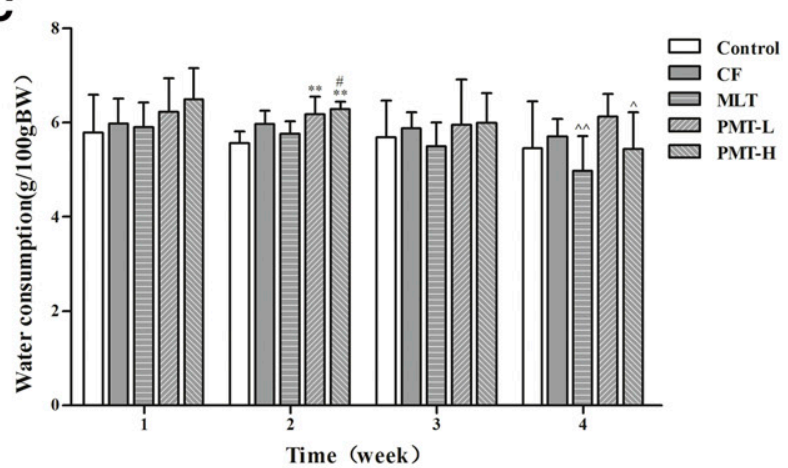

$\mathbf{E}$

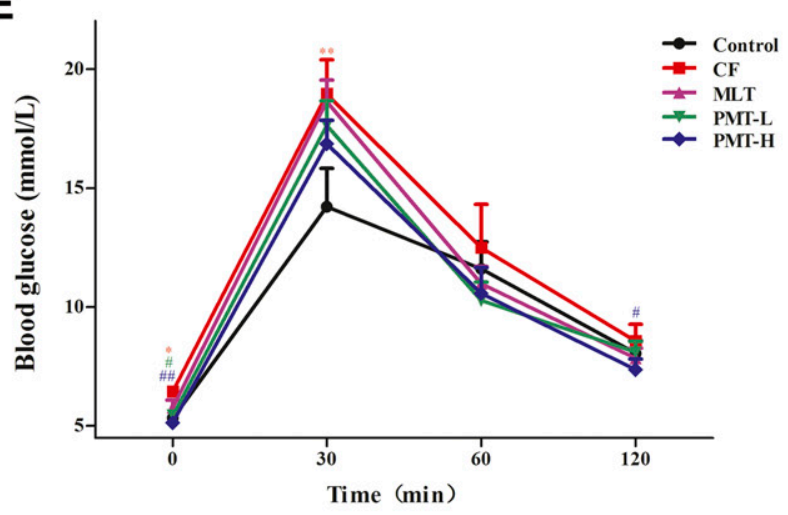

G

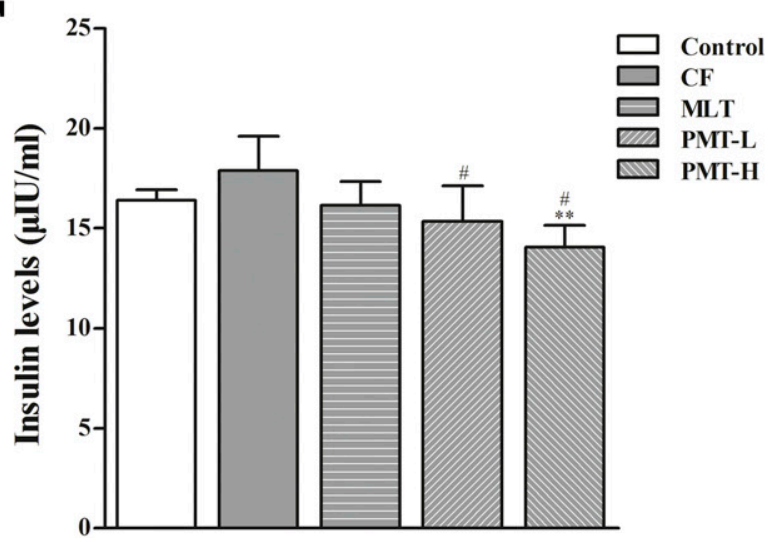

B

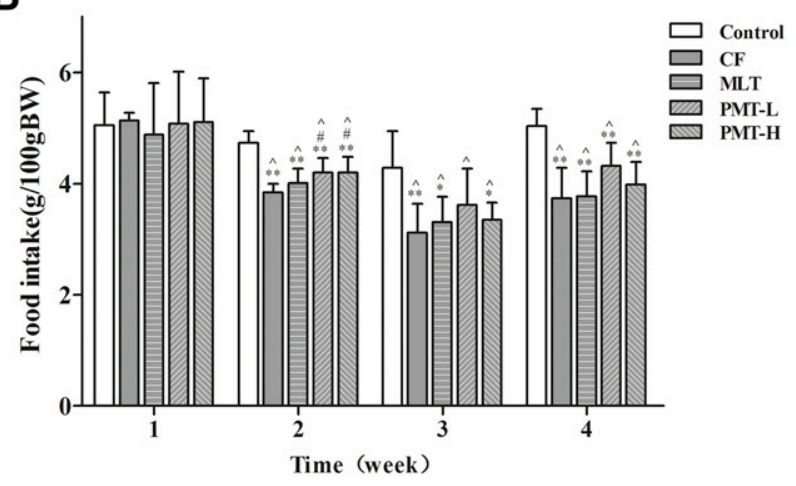

D

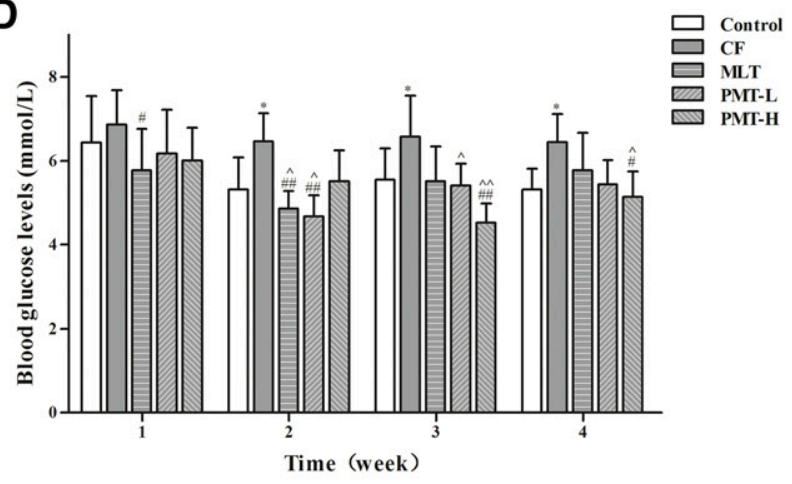

$\mathbf{F}$

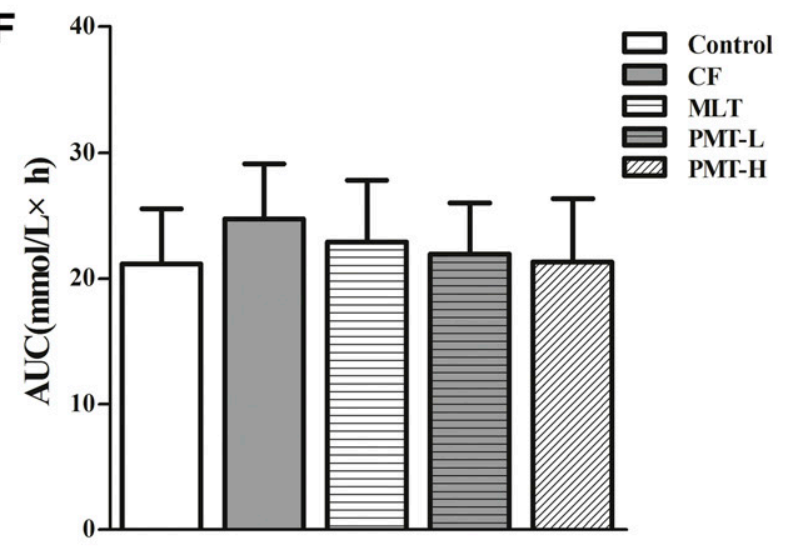

H

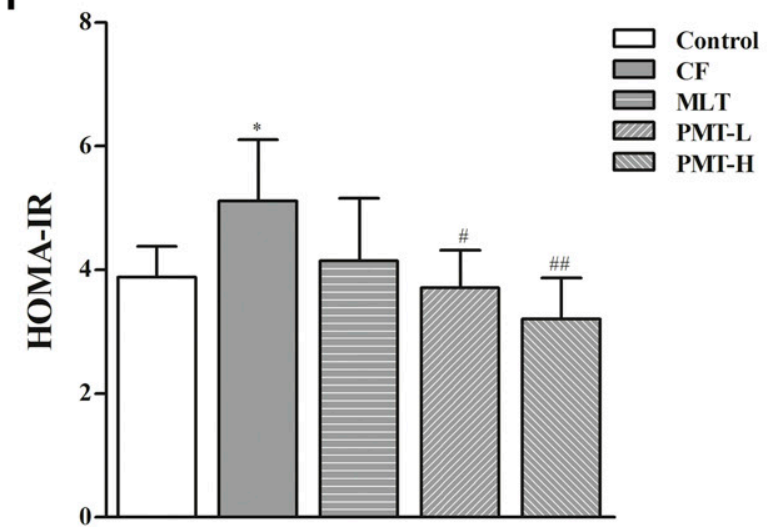




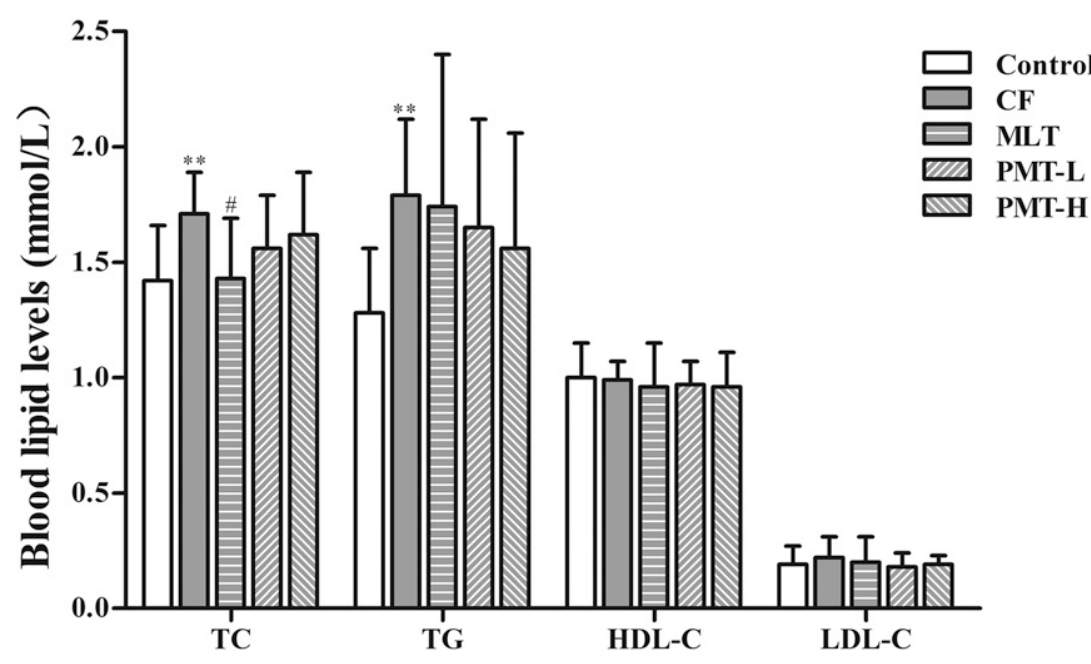

Fig. 4. Effects of piromelatine on blood lipid levels over 4 weeks of treatment. The values are expressed as the means \pm S.D. $(n=8-10$ per group $) * * P<0.01$ vs. control group, ${ }^{\#} P<0.05$ vs. $C F$ group via one-way analysis of variance with Dunnett's post-hoc analyses.

in adipose tissue that might potentially ameliorate the effect of $\mathrm{CF}$ on the HPA axis via such actions as diminished active CORT secretion and increased sensitivity to GC feedback (Houdek et al., 2015).

Effect of Piromelatine on Adipose Gene Expression in CF Rats. Adipose tissue is a complex endocrine and immune organ that responds to and, in turn, releases signals that represent or reflect metabolic risk factors (Kershaw and Flier, 2004). To further examine the changes in gene expression mediated by piromelatine treatment, we evaluated the expression of molecules involved in glucose and lipid metabolism in adipose tissue in CF rats. Upon IR induction, the relative mRNA expression of adiponectin, glucose transporter type-4 (GLUT-4), and PPAR- $\gamma$ was decreased by $57.00 \%$, 44.00\%, and $39.00 \%$, respectively (all $P<0.01$ ) (Fig. 6, A, F, and G), and the relative mRNA expression of leptin, TNF- $\alpha$, and IL-6 was increased by $17.00 \%, 64.00 \%$ $(P<0.01$ ), and $14.00 \%$, respectively (Fig. 6, B, D, and E). The leptin/adiponectin ratio was increased by $64.00 \%(P<0.01)$ (Fig. 6C).

Thus, piromelatine caused dose-dependent increases in the relative expression of adiponectin and dose-dependent decreases in leptin expression, with both tested doses resulting in statistically significant changes. MLT treatment also mediated slight changes in the expression of adiponectin and leptin. Adiponectin exerts anti-inflammatory and antidiabetic effects (Zhao et al., 2015), and leptin represents one of the key peripheral cues that signals the status of body energy reserves to the brain (Burgos-Ramos et al., 2012). These results imply that several improvements in T2DM symptoms are potentially regulated at the transcript level by piromelatine.

Additionally, the expression of the proinflammatory cytokines TNF- $\alpha$ and IL-6 increased significantly in CF rats compared with normal rats. However, the administration of piromelatine potentially reversed the changes in inflammatory markers caused by chronic stress and an HFD. TNF- $\alpha$ and IL-6 induce IR, promote lipolysis, and reduce adipogenesis (Hajri et al., 2011). These results might further support the potentially beneficial effects of MLT and piromelatine on glucose homeostasis and insulin sensitivity. Piromelatine treatment also mediated slight changes in the expression of GLUT-4, but there were no significant differences.

Effect of Piromelatine on Adipose GR and 11 $\beta$ HSD1 Protein Expression in CF Rats. To further examine the changes in HPA-axis regulation, adipose GR and $11 \beta \mathrm{HSD} 1$ protein expression was analyzed by Western blotting. Compared with the control group, the protein level of GR was decreased in the CF group, whereas treatment with MLT and piromelatine attenuated this suppression $(P<0.01) 11 \beta H S D 1$ is a possible link between GC biology and the development of IR. After induction of IR for 4 weeks, $11 \beta$ HSD1 expression in adipose tissue was markedly increased $(P<0.01)$. The higher protein levels were significantly reduced in CF rats by $44.24 \%$ $(P<0.01), 50.91 \%(P<0.01)$, and $61.82 \%(P<0.01)$ after MLT or low- or high-dose piromelatine treatment, respectively. These results verify the protective effects of MLT and piromelatine on HPA-axis function at the molecular level (Fig. 7).

\section{Discussion}

Daily exposure to stress and consumption of high-caloric food rich in fat, which are features of a modern lifestyle, are correlated with an increased incidence of metabolic syndrome, T2DM, and cardiovascular disease (Kovačević et al., 2016). An HFD has been shown to impair insulin sensitivity and lead to IR (Tanaka et al., 2007; Flanagan et al., 2008). Furthermore, chronic-stress-induced excessive GC production is known to result in whole-body IR and impaired glucose homeostasis, which have been suggested as triggers of

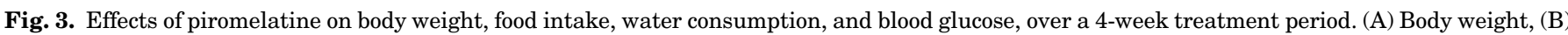

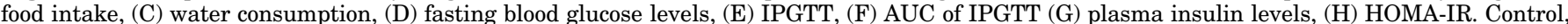

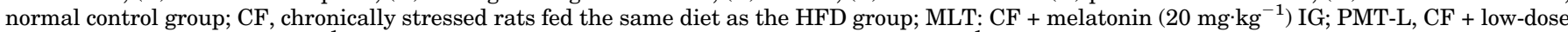

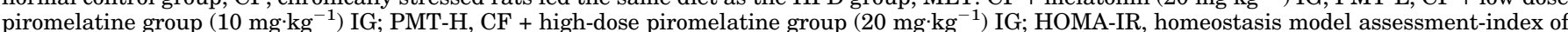

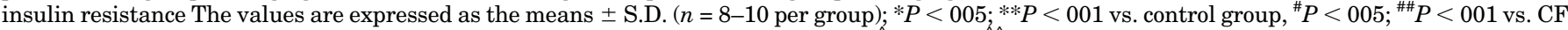

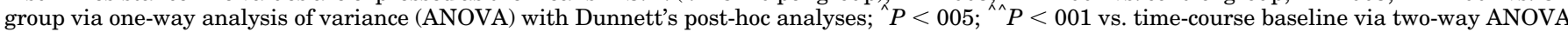
with Bonferroni post-hoc analyses. 
A

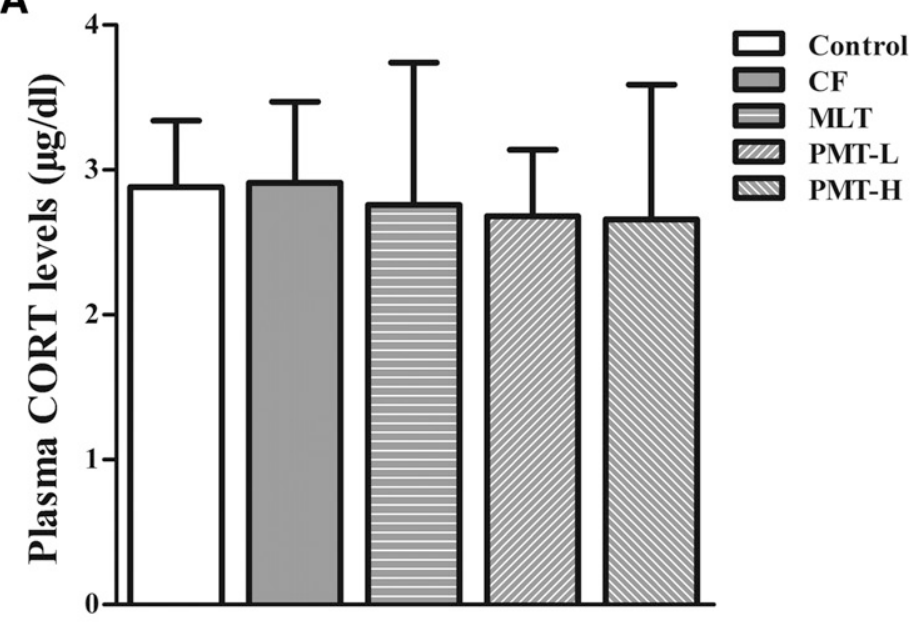

B

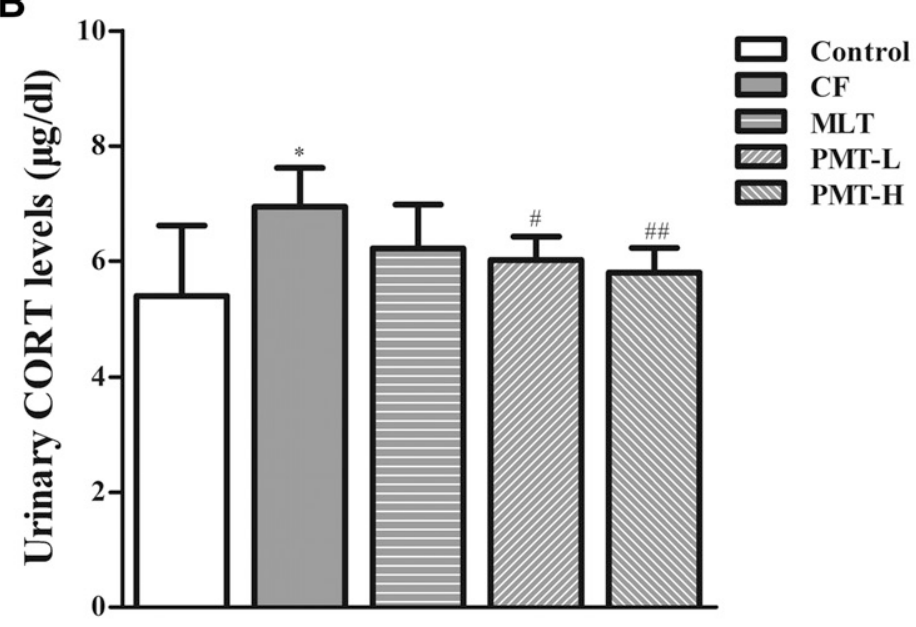

Fig. 5. Effects of piromelatine and MLT on HPA-axis function over 4 weeks of treatment. (A) Plasma CORT levels and (B) urinary CORT levels. (C) The relative expression of GR and $11 \beta \mathrm{HSD} 1 \mathrm{mRNA}$ in adipose tissue was measured through a real-time RT-qPCR analysis. Values are expressed as the means \pm S.D. $(n=8-10$ per group); ${ }^{*} P<005 ; * * P<001$ vs. control group; ${ }^{*} P<005$; ${ }_{\# \#} P<001$ vs. CF group via one-way analysis of variance with Dunnett's post-hoc analyses.

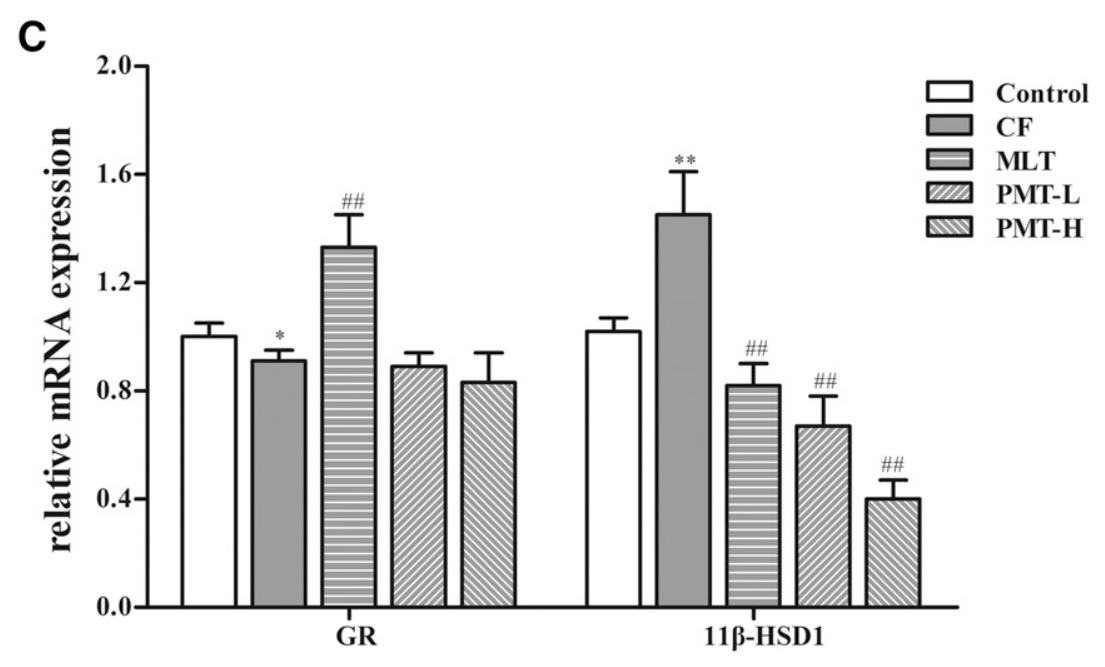

diabetes (Novak et al., 2013; Geer et al., 2014). In humans, prolonged chronic social stress is associated with higher cortisol levels, increased abdominal fat deposition, hyperglycemia, and IR (Epel et al., 2001; Aschbacher et al., 2014). The results obtained in experiment I demonstrate that the combination of an HFD and chronic stress impairs glucose metabolism and induces IR (as demonstrated by the IPGTT, ITT, and HOMA-IR index), with a loss of body weight gain and the gradual onset of symptoms of hyperglycemia (Zhang et al., 2014), which is in accordance with previous studies (Zardooz et al., 2006; Bruder-Nascimento et al., 2013; Kovačević et al., 2016; Zemdegs et al., 2016). 
A

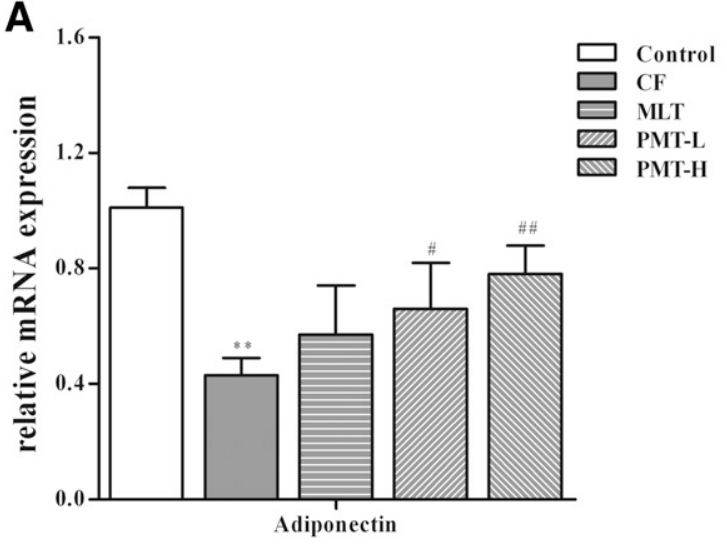

C

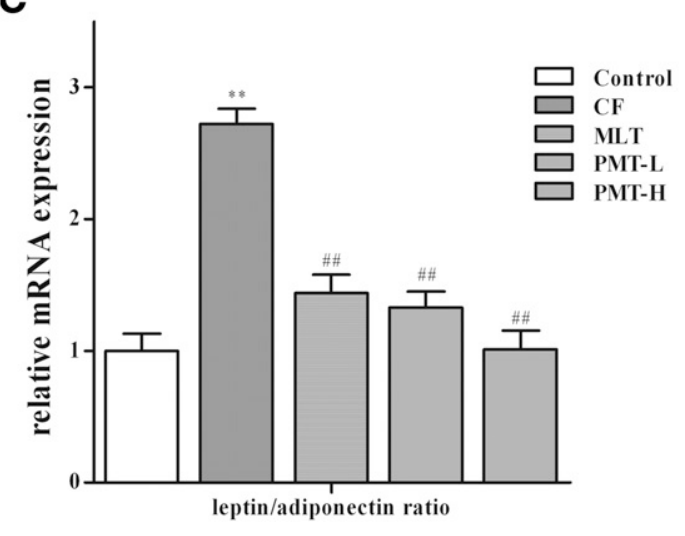

E

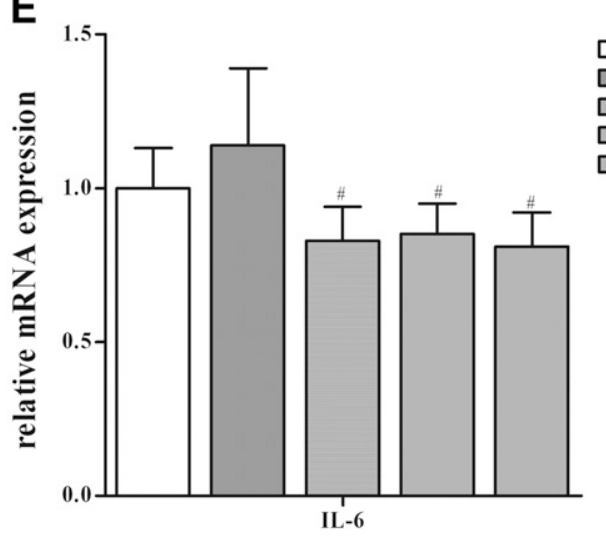

Control

CF PMT-L

PNT-H
PNT-L
B

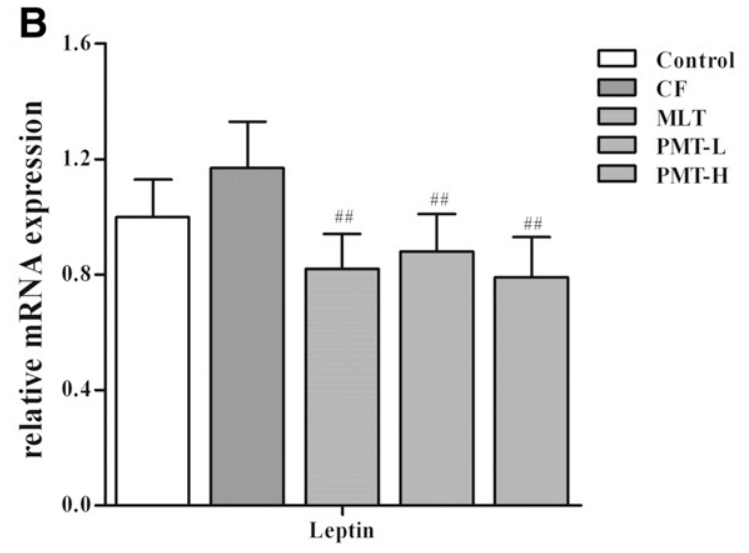

D

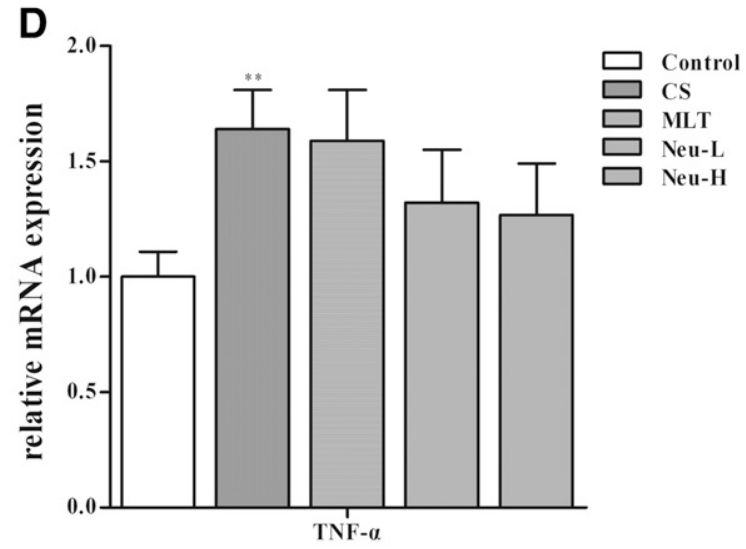

F

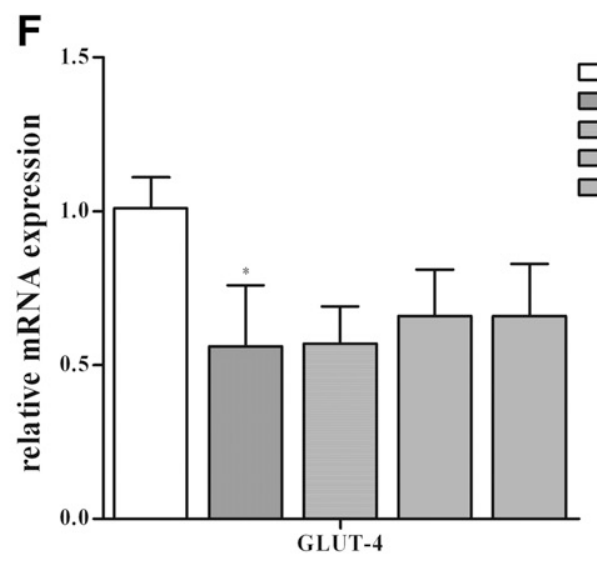

G
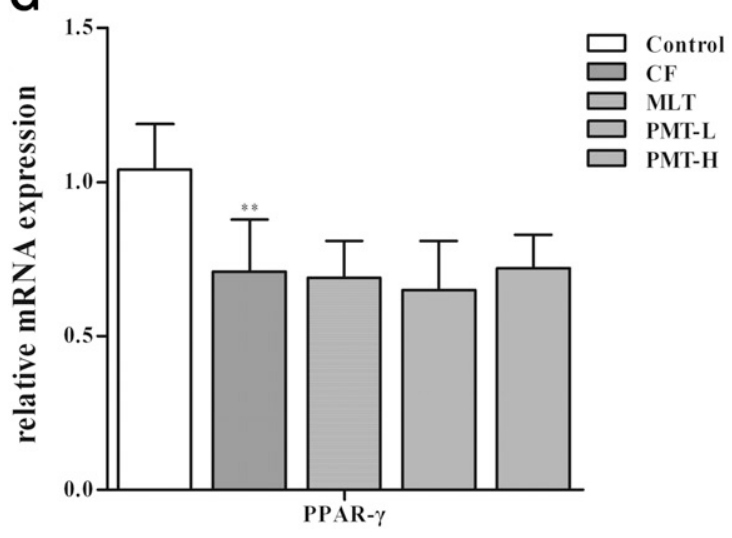

PMT-L 

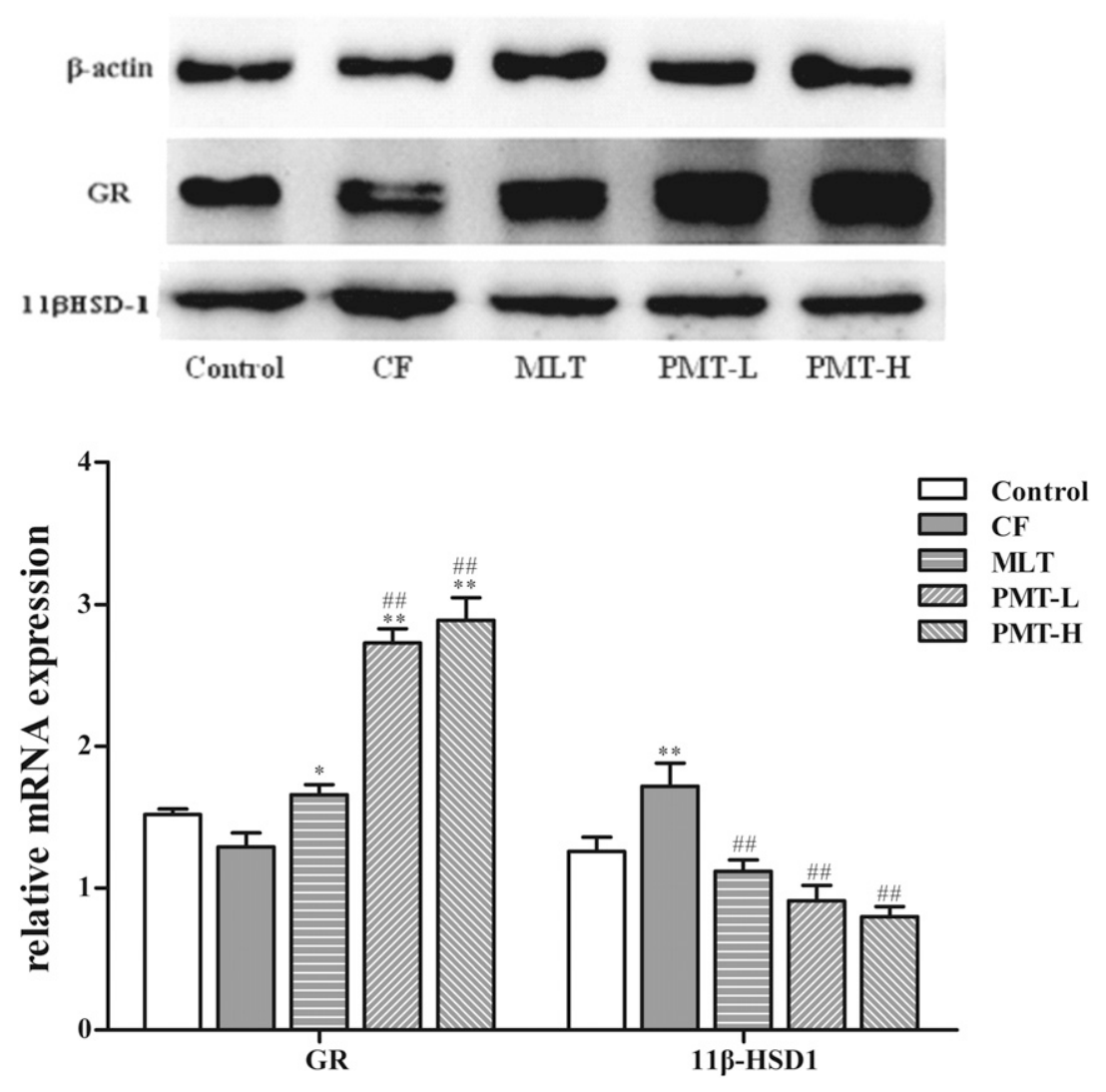

Fig. 7. Effects of piromelatine and MLT on adipose GR and $11 \beta$ HSD-1 protein expression in CF rats over 4 weeks of treatment. Relative expression of the GR and $11 \beta \mathrm{HSD}-$ 1 proteins in adipose tissue was measured by Western blotting. Band intensities were quantified by densitometry. Values are expressed as the means \pm S.D. $(n=8-10$ per group); $* P<005$; $* * P<001$ vs. control group; ${ }^{\#} P<$ $005 ;{ }^{\# \#} P<001$ vs. $\mathrm{CF}$ group via one-way analysis of variance with Dunnett's post-hoc analyses.

In experiment II, there was a slight increase in appetite in the first week and a significant reduction in food intake after 1 week in the CF group, and a suppression of body weight gain was also observed in the $\mathrm{CF}$ rats $(P<0.05$ vs. the control group), indicating a time-dependent interplay between the HFD and stress. Short-term stress might increase the intake of highly palatable, energy-dense foods (Finger et al., 2012), whereas long-term stress exposure could inhibit an increase in weight gain and caloric intake (Marti et al., 1994), suggesting that chronic exposure to stress prevents the metabolic effects of an HFD on energy intake (Finger et al., 2012).

In clinical practice, psychologic stress might cause some people to eat less but encourage others to eat more (Stone and Brownell, 1994), and chronic stress has been shown to cause both weight gain and weight loss (Nyberg et al., 2012). The associations between stress and eating and between stress and weight remain challenging owing to the complexity of eating and body weight regulation. Eating and body weight are regulated by a number of factors, including gender, education, body mass index, the energetic state of the organism, gastrointestinal hormonal and neuronal signals, nutrients, free time during the day, food availability, and food quality choice. In addition to these factors, disease state, genetics and ethnic differences also cause interindividual differences in the responses of humans to stress (Iwasaki et al., 2017).
The animal model established in our present study represents a mechanistic model that incorporates most of the factors observed in humans and was used to analyze treatments for impaired glucose metabolism induced by chronic stress and an HFD.

There are clear gender differences in the responses to stressful daily problems, including eating (Stone and Brownell, 1994), body weight (Darling et al., 2017), hormonal regulation and other physiologic and pathologic outcomes (Fachin et al., 2008; Deak et al., 2015). In humans, the relationship between chronic life stress and weight gain is stronger in males than in females (Torres and Nowson, 2007). In terms of HPA-axis regulation, female rats exhibit higher GC levels and slower GC elimination after HPA-axis activation (Paris et al., 2010; Babb et al., 2013). Another study demonstrated that estrogen impairs GC-dependent HPA-axis negative feedback via estrogen receptor $\alpha$ within the hypothalamus (Weiser and Handa, 2009). A large number of studies use male rats to model stress (Zardooz et al., 2006; Detanico et al., 2009; Bruder-Nascimento et al., 2013; Zhang et al., 2014; Kovačević et al., 2016; Zemdegs et al., 2016); therefore, males have been investigated more often in studies of stress.

We demonstrated that piromelatine and MLT improved the dysfunction of glucose and lipid metabolism and insulin sensitivity in rats with IR induced by an HFD combined with

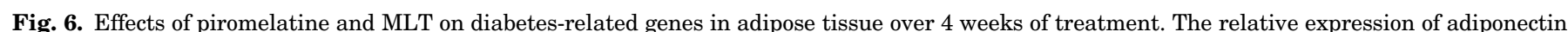

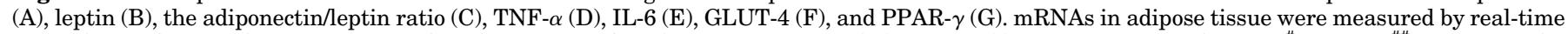

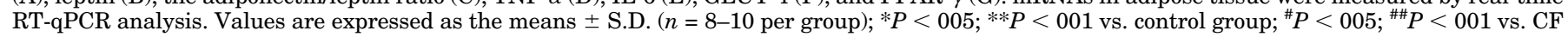
group via one-way analysis of variance with Dunnett's post-hoc analyses. 
chronic stress. Furthermore, mechanistic experiments showed that piromelatine administration effectively alleviated HPA-axis hyperfunctioning and potentially restored the expression of several key genes that were impaired by an HFD and chronic stress exposure. Our results thus suggest the potential therapeutic effects of MLT and piromelatine on IR.

The HPA axis is regulated by GR and is responsible for the stimulation of adrenal corticosteroids required to maintain homeostasis in response to stress (Gupta et al., 2007). The plasma CORT level measured in this study, which represents the concentration of total CORT (free and bound) in circulation (Priyadarshini and Anuradha, 2016), remained unchanged in $\mathrm{CF}$ animals compared with control animals, although the $\mathrm{CF}$ rats did exhibit significantly elevated levels of urinary CORT, which reflects the average amount of free and biologically active CORT in the body. In addition, we observed increased levels of $11 \beta$ HSD1, which is a possible link between GC biology and the development of IR (Stimson and Walker, 2013). Both animal and human studies have indicated that metabolic disturbances are accompanied by increased intracellular $11 \beta$ HSD1-driven regeneration of CORT (Kovačević et al., 2016). Decreased GR mRNA expression has been investigated in CF rats because it plays an important role in modulating GC feedback on the HPA axis (Moisan et al., 1990) in CF rats. CORT binds to GRs in the paraventricular nucleus of hypothalamus, pituitary, and other central nervous system sites in the forebrain to mediate the negative feedback inhibition of CORT (Keller-Wood, 2015). Chronic psychosocial stress leads to increased HPA-axis activity and impaired GR function, which is associated with hypercortisolemia and reduced GR-mediated negative feedback on the HPA axis (De Kloet et al., 1998). High concentrations of GRs enhance CORT negative feedback on the hypothalamus, thereby forcing the HPA axis into an alternative, low CORT state (Gupta et al., 2007).

Thus, decreased GR biosynthesis and elevated free CORT production are indicative of impaired functioning of the HPAaxis feedback mechanisms (Blanchard et al., 1995; Konakchieva et al., 1998). Our results therefore suggest an impairment of HPA regulation, which is consistent with previous reports (Bruder-Nascimento et al., 2013; Kovačević et al., 2016).

Recently, MLT has received attention as a potential chronotherapeutic drug for metabolic disease. Evidence suggests that MLT, a neurohormone that is secreted from the pineal gland, might normalize CORT secretion or the activity of the HPA axis (Detanico et al., 2009). Increasing evidence suggests that MLT plays an important role in glucose metabolism in both animals and humans (Peschke and Mühlbauer, 2010; Agil et al., 2012). In the present study, MLT and piromelatine enhanced GR expression and inhibited $11 \beta$ HSD1 expression, reflecting an enhanced negative feedback on the HPA axis and decreased active CORT secretion, which would result in a reduction of the active free GCs in circulation. This finding suggests that piromelatine exerts a strong normalizing effect on HPA-axis hyperfunctioning, thus ameliorating IR, lowering fasting plasma glucose levels, and improving other metabolic dysfunction symptoms induced by chronic stress and an HFD (Kovačević et al., 2016). Our data showed that MLT and piromelatine treatment improved glucose tolerance in $\mathrm{CF}$ rats, as demonstrated by decreased blood glucose levels after glucose load in an IPGTT and a decreased HOMA-IR index. Notably, these results are similar to those obtained in another study, one in which the administration of piromelatine improved insulin sensitivity in sleep-deprived rats (She et al., 2009). This result further supports the beneficial effects of piromelatine on insulin sensitivity.

GCs (hyperglycemic effect) and insulin (hypoglycemic effect) play important roles in energetic balance, and this balance can be impaired by exposure to chronic stress (Bruder-Nascimento et al., 2013). GCs exert diabetogenic effects that interfere with insulin action at different levels, causing, for example, impaired regulation of energy homoeostasis through leptin and adiponectin (Lee and Shao., 2014; Marangon et al., 2014; Isorna et al., 2017), disruptions in glucose transport to intracellular spaces through GLUT4 (Coderre et al., 1996), and inhibition of the potent insulin sensitizer PPAR (Rosen and Spiegelman, 2001). We observed several changes in the expression of genes that possibly regulate glucose and lipid metabolism after MLT and piromelatine administration, although a direct assessment of blood lipid levels did not show significant differences.

Adiponectin is an adipokine with anti-inflammatory and antidiabetic properties. Hypoadiponectinemia is closely associated with endothelial dysfunction and IR in obesity and diabetes (Zhao et al., 2015). The adipose tissue-derived hormone leptin has a well-characterized role in energy homoeostasis and modulates appetite, food intake, body weight, adipose stores, and immune functions (BurgosRamos et al., 2012). Both adiponectin and leptin are regulated by GCs and in turn might impair insulin sensitivity (CastanLaurell et al., 2011). As a potent insulin sensitizer, adipocyte PPAR $\gamma$ increases the expression of genes that promote triacylglycerol storage and induce lipolysis, thus decreasing ectopic lipid deposition (Rosen and Spiegelman, 2001; Berthiaume et al., 2004). GLUT-4 in adipose tissue transports glucose into fat cells, which is a rate-determining step for glucose consumption in adipocytes (Coderre et al., 1996).

Our data demonstrated that CF decreases PPAR $\gamma$, GLUT-4 and adiponectin expression and increases leptin expression at the transcriptional level, which is partly consistent with previous findings (Bruder-Nascimento et al., 2013; Kovačević et al., 2016). Our results also suggest that piromelatine relieves the suppression of adiponectin expression and the overexpression of leptin but has no effect on PPAR $\gamma$ or GLUT-4 mRNA expression, demonstrating that piromelatine might potentially promote energy homoeostasis and improve whole-body IR but does not affect adipogenesis or lipid disposal, which is supported by the observation that the blood lipid levels did not change. Thus, the effects of piromelatine on the expression of several genes could possibly contribute to their ability to regulate glucose homeostasis.

Low-grade inflammation has been linked to risk of T2DM and atherosclerotic vascular diseases (Uusitupa and Schwab, 2013). In response to excess adiposity and macrophage infiltration in adipose tissue, numerous proinflammatory cytokines, including TNF- $\alpha$ and IL-6, are concomitantly increased in adipose tissue, and their levels correlate with the degree of IR (Kern et al., 2001; Galic et al., 2010). The increased levels of TNF- $\alpha$ and IL- 6 gene expression observed in the CF group might indicate the presence of low-grade inflammation in chronically stressed rats fed an HFD. MLT has been reported to reduce inflammatory injury by downregulating proinflammatory molecules, e.g., TNF- $\alpha$ and IL-6, in an nuclear factor 
$\kappa$ B-dependent manner in young male Zucker diabetic fatty rats or in rats fed an HFD (Agil et al., 2013; Cano Barquilla et al., 2014; Cutando et al., 2015). In our study, piromelatine administration appeared to reverse the increases in proinflammatory cytokines in the adipose tissues of rats subjected to $\mathrm{CF}$, indicating the potential alleviation of inflammation and IR.

In recent years, the beneficial effects of MLT in experimental or even clinical conditions of obesity and metabolic syndrome have been demonstrated, but its therapeutic use has been limited by a short half-life (0.5 hours) (Paulis et al., 2012), and the possibility that administration of higher doses of MLT might desensitize MLT receptors (Gerdin et al., 2004). Piromelatine was developed to extend the half-life of MLT to up to 1.5-3.0 hours, leading to a prolonged duration of action and greater efficiency than other MLT supplements.

MLT appears to have an approximately 4.7-fold higher affinity for the MT1 receptor [dissociation constancy for binding affinity $\left(K_{\mathrm{i}}\right)=0.081 \mathrm{nM}$ ] than for the MT2 receptor $\left(K_{\mathrm{i}}=0.383 \mathrm{nM}\right)$. Piromelatine shows a similar binding affinity to both types of MLT receptors [binding affinity $\mathrm{MT} 1\left(K_{\mathrm{i}}=\right.$ $22 \mathrm{nM})$, binding affinity MT2 $\left.\left(K_{\mathrm{i}}=34 \mathrm{nM}\right)\right]$ (Yalkinoglu et al., 2010). These differences between MLT and piromelatine might explain why the similar MT1 and MT2 agonistic activities of piromelatine exert an improved effect on metabolism in CF rats.

Many of MLT's actions are mediated through its direct interactions with MT1 and MT2, which are G protein-coupled membrane-bound receptors (Masana et al., 2002), or its indirect actions via nuclear orphan receptors from the retinoid orphan receptors/retinoid $\mathrm{Z}$ receptors (ROR/RZR) family, which are MLT nuclear receptors functioning as transcription factors (Becker-Andre et al., 1994; Wiesenberg et al., 1995). MLT also binds to the quinone reductase II (QR2) enzyme, previously defined the MT3 receptor, with a low affinity (Mailliet et al., 2004). Melatonin also acts through nonreceptor-mediated mechanisms, for example serving as a scavenger for reactive oxygen species and reactive nitrogen species (Reiter et al., 2001, 2007) and as a broad-spectrum antioxidant (Koc et al., 2003; Tomás-Zapico and Coto-Montes, 2005).

Piromelatine selectively binds the melatonergic MT1 and MT2 receptors over QR2/MT3 and ROR/RZR, which suggests crucial roles for only MT1 and MT2 receptors in neuroendocrine mechanisms, such as normalization of HPA-axis and glucose metabolism (Torres-Farfan et al., 2003; Zhou et al., 2017). In contrast, the binding of MLT additionally to either the QR2/MT3 or ROR/RZR possibly has the opposite effects on insulin levels and the mRNA expression of GR and other numerous genes. There is much evidence to support our hypothesis, the overexpression of $\mathrm{ROR} \alpha$ or treatment with an ROR $\alpha$-specific agonist enhances the expressions of inflammatory cytokines and increases the number of infiltrated macrophages into adipose tissue, whereas adipose tissue inflammation has been linked to metabolic diseases such as obesity and T2DM (Liu et al., 2017). The effects of MLT and its agonists on diabetes through QR2/MT3 and ROR/RZR are worth additional research.

Additionally, food rich in energy leads to more extra fat stored in the body, finally resulting in obesity. Melatonin and melatonin agonists induce extra energy expenditure and limit obesity in rodents without affecting food intake or having a thermogenic effect on brown adipose tissue (BAT), such as stimulating growth of BAT and increasing BAT activity (Tan et al., 2011; Jiménez-Aranda et al., 2013). These important factors contributed to the effect of melatonin and melatonin agonists on obesity treatment.

In summary, our results clearly demonstrate that $\mathrm{CF}$ exposure induces IR as well as changes in free CORT levels and HPA-axis hyperfunctioning. Piromelatine administration effectively ameliorates glucose intolerance in an IPGTT and insulin insensitivity in the HOMA-IR index as well as the associated metabolic deficits in CF rats. Mechanistic experiments indicated that the effects of piromelatine might be related to decreases in the free CORT level and 11 HHSD1 expression and the regulation of glucose metabolism-related genes. Moreover, the suppression of proinflammatory TNF- $\alpha$ and IL- 6 expression in adipose tissue also possibly mediates the insulin sensitivity effect in CF rats. We conclude that piromelatine might be a potential drug for the treatment of glucose intolerance and IR. However, more studies are necessary to better understand the mechanisms of action of piromelatine on metabolic disturbance.

\section{Acknowledgments}

The authors thank our laboratory members for their valuable suggestions and technical assistance.

\section{Authorship Contributions}

Participated in research design: Zhou, Zhang, Jia.

Conducted experiments: Zhou, Wang, Luo, Jia.

Performed data analyses: Zhou, Wang, Li, Zhang, Jia.

Wrote or contributed to the writing of the manuscript: Zhou, Zhang, Jia, Laudon.

\section{References}

Agil A, Reiter RJ, Jiménez-Aranda A, Ibán-Arias R, Navarro-Alarcón M, Marchal JA, Adem A, and Fernández-Vázquez G (2013) Melatonin ameliorates low-grade inflammation and oxidative stress in young Zucker diabetic fatty rats. $J$ Pineal Res 54:381-388.

Agil ARosado I, Ruiz R, Figueroa A, Zen N, and Fernández-Vázquez G (2012) Melatonin improves glucose homeostasis in young Zucker diabetic fatty rats. J Pineal Res 52:203-210.

Aschbacher K, Kornfeld S, Picard M, Puterman E, Havel PJ, Stanhope K, Lustig RH, and Epel E (2014) Chronic stress increases vulnerability to diet-related abdominal fat, oxidative stress, and metabolic risk. Psychoneuroendocrinology 46:14-22.

Babb JA, Masini CV, Day HEW, and Campeau S (2013) Sex differences in activated corticotropin-releasing factor neurons within stress-related neurocircuitry and hypothalamic-pituitary-adrenocortical axis hormones following restraint in rats. Neuroscience 234:40-52.

Becker-André M, Wiesenberg I, Schaeren-Wiemers N, André E, Missbach M, Saurat JH, and Carlberg C (1994) Pineal gland hormone melatonin binds and activates an orphan of the nuclear receptor superfamily. J Biol Chem 269:28531-28534.

Berthiaume M, Sell H, Lalonde J, Gélinas Y, Tchernof A, Richard D, and Deshaies Y (2004) Actions of PPARgamma agonism on adipose tissue remodeling, insulin sensitivity, and lipemia in absence of glucocorticoids. Am J Physiol Regul Integr Comp Physiol 287:R1116-R1123.

Björntorp P (2001) Do stress reactions cause abdominal obesity and comorbidities? Obes Rev 2:73-86.

Björntorp P and Rosmond R (1999) Hypothalamic origin of the metabolic syndrome X. Ann N Y Acad Sci 892:297-307.

Björntorp P and Rosmond R (2000) The metabolic syndrome-a neuroendocrine disorder? Br J Nutr 83 (Suppl 1):S49-S57.

Blanchard DC, Spencer RL, Weiss SM, Blanchard RJ, McEwen B, and Sakai RR (1995) Visible burrow system as a model of chronic social stress: behavioral and neuroendocrine correlates. Psychoneuroendocrinology 20:117-134.

Bruder-Nascimento T, Campos DH, Alves C, Thomaz S, Cicogna AC, and Cordellini S (2013) Effects of chronic stress and high-fat diet on metabolic and nutritional parameters in Wistar rats. Arq Bras Endocrinol Metabol 57:642-649.

Buendia I, Gómez-Rangel V, González-Lafuente L, Parada E, León R, Gameiro I, Michalska P, Laudon M, Egea J, and López MG (2015) Neuroprotective mechanism of the novel melatonin derivative Neu-P11 in brain ischemia related models. Neuropharmacology 99:187-195.

Burgos-Ramos E, Canelles S, Perianes-Cachero A, Arilla-Ferreiro E, Argente J, and Barrios V (2012) Adipose tissue promotes a serum cytokine profile related to lower insulin sensitivity after chronic central leptin infusion. PLoS One 7:e46893.

Cano Barquilla P, Pagano ES, Jiménez-Ortega V, Fernández-Mateos P, Esquifino AI and Cardinali DP (2014) Melatonin normalizes clinical and biochemical 
parameters of mild inflammation in diet-induced metabolic syndrome in rats. $J$ Pineal Res 57:280-290.

Carocci A, Catalano A, and Sinicropi MS (2014) Melatonergic drugs in development. Clin Pharmacol 6:127-137.

Castan-Laurell I, Dray C, Attané C, Duparc T, Knauf C, and Valet P (2011) Apelin, diabetes, and obesity. Endocrine 40:1-9.

Coderre L, Vallega GA, Pilch PF, and Chipkin SR (1996) In vivo effects of dexamethasone and sucrose on glucose transport (GLUT-4) protein tissue distribution. Am J Physiol 271:E643-E648.

Cutando A, Montero J, Gómez-de Diego R, Ferrera MJ, and Lopez-Valverde A (2015) Effect of topical application of melatonin on serum levels of C-reactive protein (CRP), interleukin-6 (IL-6) and tumor necrosis factor-alpha (TNF- $\alpha$ ) in patients with type 1 or type 2 diabetes and periodontal disease. J Clin Exp Dent 7: e628-e633.

Darling KE, Fahrenkamp AJ, Wilson SM, Karazsia BT, and Sato AF (2017) Does social support buffer the association between stress eating and weight gain during the transition to college? Differences by gender. Behav Modif 41:368-381.

Deak T, Quinn M, Cidlowski JA, Victoria NC, Murphy AZ, and Sheridan JF (2015) Neuroimmune mechanisms of stress: sex differences, developmental plasticity, and implications for pharmacotherapy of stress-related disease. Stress 18:367-380.

De Kloet ER, Vreugdenhil E, Oitzl MS, and Joëls M (1998) Brain corticosteroid receptor balance in health and disease. Endocr Rev 19:269-301.

Detanico BC, Piato AL, Freitas JJ, Lhullier FL, Hidalgo MP, Caumo W, and Elisabetsky E (2009) Antidepressant-like effects of melatonin in the mouse chronic mild stress model. Eur J Pharmacol 607:121-125.

Dinneen S, Alzaid A, Miles J, and Rizza R (1993) Metabolic effects of the nocturnal rise in cortisol on carbohydrate metabolism in normal humans. J Clin Invest 92 2283-2290.

Epel E, Lapidus R, McEwen B, and Brownell K (2001) Stress may add bite to appetite in women: a laboratory study of stress-induced cortisol and eating behavior. Psychoneuroendocrinology 26:37-49.

Fachin A, Silva RK, Noschang CG, Pettenuzzo L, Bertinetti L, Billodre MN, Peres W, Busnello F, and Dalmaz C (2008) Stress effects on rats chronically receiving a highly palatable diet are sex-specific. Appetite 51:592-598.

Finger BC, Dinan TG, and Cryan JF (2012) The temporal impact of chronic intermittent psychosocial stress on high-fat diet-induced alterations in body weight. Psychoneuroendocrinology 37:729-741.

Flanagan AM, Brown JL, Santiago CA, Aad PY, Spicer LJ, and Spicer MT (2008) High-fat diets promote insulin resistance through cytokine gene expression in growing female rats. J Nutr Biochem 19:505-513.

Galic S, Oakhill JS, and Steinberg GR (2010) Adipose tissue as an endocrine organ. Mol Cell Endocrinol 316:129-139.

Geer EB, Islam J, and Buettner C (2014) Mechanisms of glucocorticoid-induced insulin resistance: focus on adipose tissue function and lipid metabolism. Endocrinol Metab Clin North Am 43:75-102.

Gerdin MJ, Masana MI, Rivera-Bermúdez MA, Hudson RL, Earnest DJ, Gillette MU, and Dubocovich ML (2004) Melatonin desensitizes endogenous MT2 melatonin receptors in the rat suprachiasmatic nucleus: relevance for defining the periods of sensitivity of the mammalian circadian clock to melatonin. FASEB $J \mathbf{1 8}$ 1646-1656

Gupta S, Aslakson E, Gurbaxani BM, and Vernon SD (2007) Inclusion of the glucocorticoid receptor in a hypothalamic pituitary adrenal axis model reveals bistability. Theor Biol Med Model 4:8.

Hajri T, Tao H, Wattacheril J, Marks-Shulman P, and Abumrad NN (2011) Regulation of adiponectin production by insulin: interactions with tumor necrosis factor$\alpha$ and interleukin-6. Am J Physiol Endocrinol Metab 300:E350-E360.

Hansen KB, Vilsbøll T, Bagger JI, Holst JJ, and Knop FK (2010) Reduced glucose tolerance and insulin resistance induced by steroid treatment, relative physical inactivity, and high-calorie diet impairs the incretin effect in healthy subjects. $J$ Clin Endocrinol Metab 95:3309-3317.

Houdek P, Polidarová L, Nováková M, Matějů K, Kubík Š, and Sumová A (2015) Melatonin administered during the fetal stage affects circadian clock in the suprachiasmatic nucleus but not in the liver. Dev Neurobiol 75:131-144.

Isorna E, de Pedro N, Valenciano AI, Alonso-Gómez ÁL, and Delgado MJ (2017) Interplay between the endocrine and circadian systems in fishes. $J$ Endocrinol 232 R141-R159.

Iwasaki S, Hamada T, Chisaki I, Andou T, Sano N, Furuta A, and Amano N (2017) Mechanism-based pharmacokinetic/pharmacodynamic modeling of the glucagon like peptide-1 receptor agonist exenatide to characterize its anti-obesity effects in diet-induced obese mice. J Pharmacol Exp Ther 362:441-449.

Jiménez-Aranda A, Fernández-Vázquez G, Campos D, Tassi M, Velasco-Perez L, Tan DX, Reiter RJ, and Agil A (2013) Melatonin induces browning of inguinal white adipose tissue in Zucker diabetic fatty rats. J Pineal Res 55:416-423.

Joseph JJ and Golden SH (2017) Cortisol dysregulation: the bidirectional link between stress, depression, and type 2 diabetes mellitus. Ann N Y Acad Sci 1391:20-34.

Keller-Wood M (2015) Hypothalamic-pituitary-adrenal axis-feedback control. Compr Physiol 5:1161-1182.

Kern PA, Ranganathan S, Li C, Wood L, and Ranganathan G (2001) Adipose tissue tumor necrosis factor and interleukin-6 expression in human obesity and insulin resistance. Am J Physiol Endocrinol Metab 280:E745-E751.

Kershaw EE and Flier JS (2004) Adipose tissue as an endocrine organ. $J$ Clin Endocrinol Metab 89:2548-2556.

Kitagawa A, Ohta Y, and Ohashi K (2012) Melatonin improves metabolic syndrome induced by high fructose intake in rats. J Pineal Res 52:403-413.

Koc M, Taysi S, Emin Buyukokuroglu M, and Bakan N (2003) The effect of melatonin against oxidative damage during total-body irradiation in rats. Radiat Res 160:251-255

Konakchieva R, Mitev Y, Almeida OF, and Patchev VK (1997) Chronic melatonin treatment and the hypothalamo-pituitary-adrenal axis in the rat: attenuation of the secretory response to stress and effects on hypothalamic neuropeptide content and release. Biol Cell 89:587-596.
Konakchieva R, Mitev Y, Almeida OF, and Patchev VK (1998) Chronic melatonin treatment counteracts glucocorticoid-induced dysregulation of the hypothalamicpituitary-adrenal axis in the rat. Neuroendocrinology 67:171-180.

Kopp C, Vogel E, Rettori MC, Delagrange P, and Misslin R (1999) The effects of melatonin on the behavioural disturbances induced by chronic mild stress in C3H/He mice. Behav Pharmacol 10:73-83.

Kovačević S, Nestorov J, Matić G and Elaković I (2016) Fructose and stress induce opposite effects on lipid metabolism in the visceral adipose tissue of adult female rats through glucocorticoid action. Eur J Nutr DOI: 101007/s00394-016-1251-8 [published ahead of print].

Laudon M, Urade Y, and Huang Z (2008) Neu-P11, a novel melatonin agonist: effects on sleep and EEG power spectra in rats. Sleep 31 (Suppl A):34-35.

Lee B and Shao J (2014) Adiponectin and energy homeostasis. Rev Endocr Metab Disord 15:149-156.

Liu Y, Chen Y, Zhang J, Liu Y, Zhang Y, and Su Z (2017) Retinoic acid receptorrelated orphan receptor $\alpha$ stimulates adipose tissue inflammation by modulating endoplasmic reticulum stress. J Biol Chem 292:13959-13969.

Mailliet F, Ferry G, Vella F, Thiam K, Delagrange P, and Boutin JA (2004) Organs from mice deleted for NRH:quinone oxidoreductase 2 are deprived of the melatonin binding site MT3. FEBS Lett 578:116-120.

Marangon PB, Silva LE, Rorato R, Gomiero Alves P, Antunes-Rodrigues J, and Elias LL (2014) Oestradiol modulates the effects of leptin on energy homeostasis by corticotrophin-releasing factor type 2 receptor. $J$ Neuroendocrinol 26:796-804.

Martí O, Martí J, and Armario A (1994) Effects of chronic stress on food intake in rats: influence of stressor intensity and duration of daily exposure. Physiol Behav 55:747-753.

Masana MI, Doolen S, Ersahin C, Al-Ghoul WM, Duckles SP, Dubocovich ML, and Krause DN (2002) MT(2) melatonin receptors are present and functional in rat caudal artery. J Pharmacol Exp Ther 302:1295-1302.

Matthews DR, Hosker JP, Rudenski AS, Naylor BA, Treacher DF, and Turner RC (1985) Homeostasis model assessment: insulin resistance and $\beta$-cell function from fasting plasma glucose and insulin concentrations in man. Diabetologia 28: $412-419$.

Moisan MP, Seckl JR, and Edwards CR (1990) 11 beta-hydroxysteroid dehydrogenase bioactivity and messenger RNA expression in rat forebrain: localization in hypothalamus, hippocampus, and cortex. Endocrinology 127:1450-1455

Novak M, Björck L, Giang KW, Heden-Ståhl C, Wilhelmsen L, and Rosengren A (2013) Perceived stress and incidence of Type 2 diabetes: a 35-year follow-up study of middle-aged Swedish men. Diabet Med 30:e8-e16.

Nyberg ST, Heikkilä K, Fransson EI, Alfredsson L, De Bacquer D, Bjorner JB, Bonenfant S, Borritz M, Burr H, Casini A, et al.; IPD-Work Consortium (2012) Job strain in relation to body mass index: pooled analysis of 160000 adults from 13 cohort studies. J Intern Med 272:65-73.

Paris JJ, Franco C, Sodano R, Freidenberg B, Gordis E, Anderson DA, Forsyth JP, Wulfert E, and Frye CA (2010) Sex differences in salivary cortisol in response to acute stressors among healthy participants, in recreational or pathological gamblers, and in those with posttraumatic stress disorder. Horm Behav 57:35-45.

Patterson ZR and Abizaid A (2013) Stress induced obesity: lessons from rodent models of stress. Front Neurosci 7:130.

Paulis L, Simko F, and Laudon M (2012) Cardiovascular effects of melatonin receptor agonists. Expert Opin Investig Drugs 21:1661-1678.

Peschke E and Mühlbauer E (2010) New evidence for a role of melatonin in glucose regulation. Best Pract Res Clin Endocrinol Metab 24:829-841.

Priyadarshini E and Anuradha CV (2017) Glucocorticoid antagonism reduces insulin resistance and associated lipid abnormalities in high-fructose-fed mice. Can $J$ Diabetes 41:41-51.

Reiter RJ, Tan DX, Manchester LC, and Qi W (2001) Biochemical reactivity of melatonin with reactive oxygen and nitrogen species: a review of the evidence. Cell Biochem Biophys 34:237-256.

Reiter RJ, Tan DX, Terron MP, Flores LJ, and Czarnocki Z (2007) Melatonin and its metabolites: new findings regarding their production and their radical scavenging actions. Acta Biochim Pol 54:1-9.

Rizza RA, Mandarino LJ, and Gerich JE (1982) Cortisol-induced insulin resistance in man: impaired suppression of glucose production and stimulation of glucose utilization due to a postreceptor detect of insulin action. J Clin Endocrinol Metab 54:131-138.

Rosen ED and Spiegelman BM (2001) PPARgamma : a nuclear regulator of metabolism, differentiation, and cell growth. J Biol Chem 276:37731-37734

Sadasivam S and Manickam A (1996) Carbohydrates, in Methods in Biochemistry, Eds. (Sadasivam S and Manickam A). pp 11-12. New Age International Pvt. Ltd. Publishers, New Delhi.

Sartori C, Dessen P, Mathieu C, Monney A, Bloch J, Nicod P, Scherrer U, and Duplain H (2009) Melatonin improves glucose homeostasis and endothelial vascular function in high-fat diet-fed insulin-resistant mice. Endocrinology 150:5311-5317.

She M, Deng X, Guo Z, Laudon M, Hu Z, Liao D, Hu X, Luo Y, Shen Q, Su Z, et al. (2009) NEU-P11, a novel melatonin agonist, inhibits weight gain and improves nsulin sensitivity in high-fat/high-sucrose-fed rats. Pharmacol Res 59.248-253.

Shieh JM, Wu HT, Cheng KC, and Cheng JT (2009) Melatonin ameliorates high fat diet-induced diabetes and stimulates glycogen synthesis via a PKCzeta-AktGSK3beta pathway in hepatic cells. J Pineal Res 47:339-344.

Siddiqui A, Madhu SV, Sharma SB, and Desai NG (2015) Endocrine stress response and risk of type 2 diabetes mellitus. Stress 18:498-506.

Stimson RH and Walker BR (2013) The role and regulation of 11ß-hydroxysteroid dehydrogenase type 1 in obesity and the metabolic syndrome. Horm Mol Biol Clin Investig 15:37-48.

Stone AA and Brownell KD (1994) The stress-eating paradox: multiple daily measurements in adult males and females. Psychol Health 9:425-436.

Stumvoll M, Goldstein BJ, and van Haeften TW (2005) Type 2 diabetes: principles of pathogenesis and therapy. Lancet 365:1333-1346.

Tan DX, Manchester LC, Fuentes-Broto L, Paredes SD, and Reiter RJ (2011) Significance and application of melatonin in the regulation of brown adipose tissue metabolism: relation to human obesity. Obes Rev 12:167-188. 
Tanaka S, Hayashi T, Toyoda T, Hamada T, Shimizu Y, Hirata M, Ebihara K, Masuzaki H, Hosoda K, Fushiki T, et al. (2007) High-fat diet impairs the effects of a single bout of endurance exercise on glucose transport and insulin sensitivity in rat skeletal muscle. Metabolism 56:1719-1728.

Tian SW, Laudon M, Han L, Gao J, Huang FL, Yang YF, and Deng HF (2010) Antidepressant- and anxiolytic effects of the novel melatonin agonist Neu-P11 in rodent models. Acta Pharmacol Sin 31:775-783.

Tirabassi G, Chelli FM, Ciommi M, Lenzi A, and Balercia G (2016) Influence of the hypothalamic-pituitary-adrenal axis dysregulation on the metabolic profile of patients affected by diabetes mellitus-associated late onset hypogonadism. Nutr Metab Cardiovasc Dis 26:53-59.

Tomás-Zapico C and Coto-Montes A (2005) A proposed mechanism to explain the stimulatory effect of melatonin on antioxidative enzymes. J Pineal Res 39:99-104.

Torres-Farfan C, Richter HG, Rojas-García P, Vergara M, Forcelledo ML, Valladares LE, Torrealba F, Valenzuela GJ, and Serón-Ferré M (2003) mt1 Melatonin receptor in the primate adrenal gland: inhibition of adrenocorticotropin-stimulated cortisol production by melatonin. J Clin Endocrinol Metab 88:450-458.

Torres SJ and Nowson CA (2007) Relationship between stress, eating behavior, and obesity. Nutrition 23:887-894.

Uusitupa M and Schwab U (2013) Diet, inflammation and prediabetes-impact of quality of diet. Can J Diabetes 37:327-331.

Weiser MJ and Handa RJ (2009) Estrogen impairs glucocorticoid dependent negative feedback on the hypothalamic-pituitary-adrenal axis via estrogen receptor alpha within the hypothalamus. Neuroscience 159:883-895.

Wiesenberg I, Missbach M, Kahlen JP, Schräder M, and Carlberg C (1995) Transcriptional activation of the nuclear receptor RZR alpha by the pineal gland hormone melatonin and identification of CGP 52608 as a synthetic ligand. Nucleic Acids Res 23:327-333.
Yalkinoglu Ö, Zisapel N, Nir T, Piechatzek R, Schorr-Neufing U, Bitterlich N, Allgaier C, Oertel R, Kluge A, and Laudon M (2010) Phase-I study of the safety, tolerability, pharmacokinetics and sleep promoting activity of Neu-P11, a novel putative insomnia drug in healthy humans (Abstract). Sleep 33:A220.

Yu J, Wei J, Ji L, and Hong X (2014) Exploration on mechanism of a new type of melatonin receptor agonist Neu-p11 in hypoxia-reoxygenation injury of myocardial cells. Cell Biochem Biophys 70:999-1003.

Zardooz H, Zahedi Asl S, Gharib Naseri MK, and Hedayati M (2006) Effect of chronic restraint stress on carbohydrate metabolism in rat. Physiol Behav 89:373-378.

Zemdegs J, Quesseveur G, Jarriault D, Pénicaud L, Fioramonti X, and Guiard BP (2016) High-fat diet-induced metabolic disorders impairs 5-HT function and anxiety-like behavior in mice. $\mathrm{Br} J$ Pharmacol 173:2095-2110.

Zhang R, Zhou J, Li M, Ma H, Qiu J, Luo X, and Jia Z (2014) Ameliorating effect and potential mechanism of Rehmannia glutinosa oligosaccharides on the impaired glucose metabolism in chronic stress rats fed with high-fat diet. Phytomedicine 21:607-614.

Zhao L, Fu Z, Wu J, Aylor KW, Barrett EJ, Cao W, and Liu Z (2015) Globular adiponectin ameliorates metabolic insulin resistance via AMPK-mediated restoration of microvascular insulin responses. J Physiol 593:4067-4079.

Zhou J, Zhang J, Luo X, Li M, Yue Y, Laudon M, Jia Z, and Zhang R (2017) Neu-P11, a novel MT1/MT2 agonist, reverses diabetes by suppressing the hypothalamicpituitary-adrenal axis in rats. Eur $J$ Pharmacol 812:225-233.

Address correspondence to: Dr. RuXue Zhang, Lanzhou General Hospital of PLA, Lanzhou 730050, PR China. E-mail: zhoujunh2016@126.com. Or, Dr. Zhengping Jia, Lanzhou General Hospital of PLA, Lanzhou 730050, PR China. E-mail: 121189539@qq.com 